\title{
ON DISCRETE SERIES SUBREPRESENTATIONS OF THE GENERALIZED PRINCIPAL SERIES
}

\author{
IVAN MATIĆ \\ University of Osijek, Croatia
}

\begin{abstract}
We study a family of the generalized principal series and obtain necessary and sufficient conditions under which the induced representation of studied form contains a discrete series subquotient. Furthermore, we show that if the generalized principal series which belongs to the studied family has a discrete series subquotient, then it has a discrete series subrepresentation.
\end{abstract}

\section{INTRODUCTION}

Generalized principal series present a particulary interesting class of the induced representations of classical $p$-adic groups. These are the representations of the form $\delta \rtimes \sigma$, where $\delta$ is an irreducible essentially squareintegrable representation of a general linear group and $\sigma$ is an irreducible square-integrable (i.e., a discrete series) representation of a classical group. Reducibility of the non-unitary generalized principal series has been fully described by Muić in [13], in terms of the Mœglin-Tadić classification of discrete series of classical $p$-adic groups $([9,10])$. It still remains to obtain a deeper insight into the composition series of reducible generalized principal series, which should have important applications in determination of the unitary dual. It is of particular interest to determine when the generalized principal series has a discrete series subquotient.

We note that the reducibility and composition series of unitary generalized principal series are an integral part of the Mœglin-Tadić classification. This important and essentially combinatorial classification now holds unconditionally due to recent Arthur's results ([1]).

2010 Mathematics Subject Classification. 22E35, 22E50, 11F70.

Key words and phrases. Discrete series, classical $p$-adic groups, Jacquet modules. 
For a strongly positive discrete series $\sigma$, complete composition series of the generalized principal series $\delta \rtimes \sigma$ have been described in [11]. We note that strongly positive discrete series have been introduced in [9] and the definition of these representations is recalled in the following section. We recall that the generalized principal series $\delta \rtimes \sigma$ is a standard representation if $\delta \cong|\operatorname{det}|_{F}^{e(\delta)} \delta^{u}$ for $\delta^{u}$ unitary and $e(\delta)>0$. Results of [11] also imply that if $\sigma$ is strongly positive and the standard representation $\delta \rtimes \sigma$ contains a discrete series subquotient, then it also contains a discrete series subrepresentation. Furthermore, if $\sigma$ is strongly positive, then every discrete series subquotient of the standard representation $\delta \rtimes \sigma$ is a subrepresentation, and the generalized principal series $\delta \rtimes \sigma$ can have at most two discrete series subrepresentations.

If $\sigma$ is a generic discrete series, then if follows from [3] that the standard representation $\delta \rtimes \sigma$ contains a discrete series subquotient if and only if it contains a discrete series subrepresentation. Methods used in that paper strongly rely on the fact that the generic representation appears in studied composition series with multiplicity one.

The aim of this paper is to extend the subrepresentation results from [11] to a larger class of generalized principal series. In the Møglin-Tadić classification, strongly positive representations serve as the basic building blocks in the construction of all discrete series, and every discrete series can be obtained by repeated adding of pairs of new and consecutive elements to the Jordan block of such representation. Thus, it is natural to start our investigation by considering a discrete series $\sigma$ obtained by adding two consecutive elements in the Jordan block of a strongly positive representation. This presents the first inductive step in the construction of discrete series and provides discrete series which are much complicated than the strongly positive ones. For instance, such representations can not be distinguished only by their sets of Jordan blocks, unlike the strongly positive ones. Also, they have played a key role in the determination of the first occurrence indices for theta lifts of discrete series of the metaplectic groups in [5].

We will study discrete series subquotients of the so-called positive generalized principal series. In this class of generalized principal series, a discrete series $\sigma$ is obtained by adding two consecutive elements in the Jordan block of a strongly positive representation, while $\delta$ assures that possible discrete series subquotients of $\delta \rtimes \sigma$ are of the same type as $\sigma$. For the precise definition of such generalized principal series, we refer the reader to the next section.

Using the Jacquet modules method, based on the structural formula of Tadić ([16]), which is a version of the Geometrical Lemma of Bernstein and Zelevinsky ([2]), we obtain the necessary and sufficient conditions under which a positive generalized principal series contains a discrete series subquotient. Then we identify a discrete series subrepresentation of each positive generalized principal series that contains a discrete series subquotient. To achieve 
this, we again rely on the Jacquet modules method, enhanced by the intertwining operators method. We examine all possible embeddings of discrete series and eliminate the inappropriate ones, using the precise determination of Jacquet modules of certain non-tempered irreducible representations. Such representations are viewed as the members of composition series described in [11]. In some cases, we find discrete series subquotients of the positive generalized principal series which do not appear as subrepresentations.

One of the main advantages of working with discrete series obtained by adding two consecutive elements in the Jordan block of an irreducible strongly positive representation is that a substantial set of their Jacquet modules can be obtained applying the structural formula of Tadic to certain well-known embeddings of such representations. Using the description of Jacquet modules of strongly positive representations given in [6], and also in [8, Section 7], the classical group parts of the Jacquet modules of investigated representations can be obtained using [11]. We note that the uniform description of such Jacquet modules can be found in [7].

At the moment, the general case of generalized principal series seems to be much more difficult and probably requires other proof techniques. However, the general case could probably be approached by our method when having a better knowledge on the Jacquet modules of general discrete series representations and of certain non-tempered representations appearing in the composition series of the generalized principal series.

We briefly describe the content of the paper, section by section.

In the following section we present some preliminaries and introduce the notion of the positive generalized principal series. In Section 3 we prove two technical results which are used several times throughout the paper. In the fourth section we obtain necessary and sufficient conditions under which the positive generalized principal series contains a discrete series subquotient, while in the fifth section for each such induced representation we explicitly describe its discrete series subrepresentation.

This work has been supported by Croatian Science Foundation under the project 9364 .

\section{NOTATION AND PRELIMINARIES}

Let $F$ denote a non-archimedean local field of the characteristic different from two. Let $J_{n}=\left(\delta_{i, n+1-j}\right)_{1 \leq i, j \leq n}$ denote an $n \times n$ matrix, where $\delta_{i, n+1-j}$ stands for the Kronecker symbol. For a square matrix $g$, we denote by $g^{t}$ (resp., $g^{\tau}$ ) the transposed matrix of $g$ (resp., the transposed matrix of $g$ with respect to the second diagonal). In what follows, we shall fix one of the series of classical groups

$$
S p(n, F)=\left\{g \in G L(2 n, F):\left(\begin{array}{cc}
0 & -J_{n} \\
J_{n} & 0
\end{array}\right) g^{t}\left(\begin{array}{cc}
0 & -J_{n} \\
J_{n} & 0
\end{array}\right)=g^{-1}\right\},
$$




$$
S O(2 n+1, F)=\left\{g \in G L(2 n+1, F): g^{\tau}=g^{-1}\right\}
$$

and denote by $G_{n}$ a rank $n$ group belonging to the series which we fixed.

A set of standard parabolic subgroups will be fixed in the usual way, i.e., in $G_{n}$ we fix a minimal $F$-parabolic subgroup consisting of upper-triangular matrices in $G_{n}$. Then the Levi factors of standard parabolic subgroups have the form $M \cong G L\left(n_{1}, F\right) \times \cdots \times G L\left(n_{k}, F\right) \times G_{n^{\prime}}$, where $G L(m, F)$ denotes the general linear group of rank $m$ over $F$ and $n_{1}+\cdots+n_{2}+n^{\prime}=n$. If $\delta_{i}$ is a representation of $G L\left(n_{i}, F\right)$ and $\tau$ a representation of $G_{n^{\prime}}$, the normalized parabolically induced representation $\operatorname{Ind}_{M}^{G_{n}}\left(\delta_{1} \otimes \cdots \otimes \delta_{k} \otimes \tau\right)$ will be denoted by $\delta_{1} \times \cdots \times \delta_{k} \rtimes \tau$. We use the similar notation to denote a parabolically induced representation of $G L(m, F)$.

By $\operatorname{Irr}\left(G_{n}\right)$ we denote the set of all irreducible admissible representations of $G_{n}$. Furthermore, let $R\left(G_{n}\right)$ denote the Grothendieck group of admissible finite length representations of $G_{n}$ and define $R(G)=\oplus_{n \geq 0} R\left(G_{n}\right)$. In a similar way we define $R(G L)=\oplus_{n \geq 0} R(G L(n, F))$.

We will also fix the notation that will be used throughout the paper.

The induced representation of the form $\delta \rtimes \sigma$, where $\delta$ is an irreducible essentially square-integrable representation of a general linear group and $\sigma$ is a discrete series representation of $G_{n}$, is called a generalized principal series.

There is a unique $e(\delta) \in \mathbb{R}$ such that $\nu^{-e(\delta)} \delta$ is unitarizable, where $\nu$ denotes the composition of the determinant mapping with the $p$-adic norm of the field $F$. If $e(\delta)>0$, the generalized principal series $\delta \rtimes \sigma$ has the unique irreducible (Langlands) quotient, which is also a unique irreducible subrepresentation of $\widetilde{\delta} \rtimes \sigma$.

By the results of [18], such representation $\delta$ is attached to a segment and we write $\delta=\delta\left(\left[\nu^{a} \rho, \nu^{b} \rho\right]\right)$, where $a, b \in \mathbb{R}$ are such that $b-a$ is a non-negative integer and $\rho$ is an irreducible unitary cuspidal representation of $G L\left(n_{\rho}, F\right)$ (this defines $\left.n_{\rho}\right)$. We recall that $\delta\left(\left[\nu^{a} \rho, \nu^{b} \rho\right]\right)$ is the unique irreducible subrepresentation of the induced representation $\nu^{b} \rho \times \nu^{b-1} \rho \times$ $\cdots \times \nu^{a} \rho$.

For $\sigma \in \operatorname{Irr}\left(G_{n}\right)$ and $1 \leq k \leq n$ we denote by $r_{(k)}(\sigma)$ the normalized Jacquet module of $\sigma$ with respect to parabolic subgroup $P_{(k)}$ having the Levi subgroup isomorphic to $G L(k, F) \times G_{n-k}$. We identify $r_{(k)}(\sigma)$ with its semisimplification in $R(G L(k, F)) \otimes R\left(G_{n-k}\right)$ and consider

$$
\mu^{*}(\sigma)=1 \otimes \sigma+\sum_{k=1}^{n} r_{(k)}(\sigma) \in R(G L) \otimes R(G),
$$

where 1 stands for the one-dimensional representation of the trivial group.

The following lemma, which has been derived in [16], presents the crucial structural formula for our calculations with Jacquet modules. 
Lemma 2.1. Let $\rho$ be an irreducible cuspidal representation of $G L(m, F)$ and $k, l \in \mathbb{R}$ be such that $k+l \in \mathbb{Z}_{\geq 0}$. Let $\sigma$ be an admissible finite length representation of $G_{n}$. Write $\mu^{*}(\sigma)=\sum_{\tau, \sigma^{\prime}} \tau \otimes \sigma^{\prime}$. Then

$$
\begin{aligned}
\mu^{*}\left(\delta\left(\left[\nu^{-k} \rho, \nu^{l} \rho\right]\right) \rtimes \sigma\right)= & \sum_{i=-k-1}^{l} \sum_{j=i}^{l} \sum_{\tau, \sigma^{\prime}} \delta\left(\left[\nu^{-i} \widetilde{\rho}, \nu^{k} \widetilde{\rho}\right]\right) \times \delta\left(\left[\nu^{j+1} \rho, \nu^{l} \rho\right]\right) \times \tau \\
& \otimes \delta\left(\left[\nu^{i+1} \rho, \nu^{j} \rho\right]\right) \rtimes \sigma^{\prime},
\end{aligned}
$$

where $\widetilde{\rho}$ denotes the contragradient of $\rho$. We omit $\delta\left(\left[\nu^{x} \rho, \nu^{y} \rho\right]\right)$ if $x>y$.

Throughout the paper we prefer to use the subrepresentation version of the Langlands classification and write the non-tempered irreducible representation $\pi$ of $G_{n}$ as a unique irreducible (Langlands) subrepresentation of the induced representation of the form $\delta_{1} \times \delta_{2} \times \cdots \times \delta_{k} \rtimes \tau$, where $\tau$ is a tempered representation of $G_{t}$ (this defines $t$ ), $\delta_{i}$ is an irreducible essentially square-integrable representation of $G L\left(n_{\delta_{i}}, F\right)$ attached to the segment $\left[\nu^{a_{i}} \rho_{i}, \nu^{b_{i}} \rho_{i}\right]$ for $i=1,2, \ldots, k$, and $a_{1}+b_{1} \leq a_{2}+b_{2} \leq \cdots \leq a_{k}+b_{k}<0$. In this case, we write $\pi=L\left(\delta_{1} \times \delta_{2} \times \cdots \times \delta_{k} \rtimes \tau\right)$.

An irreducible representation $\sigma \in R(G)$ is called strongly positive if for every embedding

$$
\sigma \hookrightarrow \nu^{s_{1}} \rho_{1} \times \nu^{s_{2}} \rho_{2} \times \cdots \times \nu^{s_{k}} \rho_{k} \rtimes \sigma_{\text {cusp }},
$$

where $\rho_{i} \in R(G L), i=1,2, \ldots, k$, are irreducible cuspidal unitary representations and $\sigma_{\text {cusp }} \in R(G)$ is an irreducible cuspidal representation, we have $\operatorname{Re}\left(s_{i}\right)>0$ for $i=1,2, \ldots, k$.

We will now recall the Mœglin-Tadić classification of discrete series for groups that we consider. Fix a certain tower of classical groups (symplectic or orthogonal). Every discrete series representation is uniquely described by three invariants: the partial cuspidal support, the Jordan block and the $\epsilon$ function.

The partial cuspidal support of a discrete series $\sigma \in \operatorname{Irr}\left(G_{n}\right)$ is an irreducible cuspidal representation $\sigma_{\text {cusp }}$ of some $G_{m}$ such that there is an irreducible admissible representation $\pi$ of $G L(n-m, F)$ such that $\sigma$ is a subrepresenation of $\pi \rtimes \sigma_{\text {cusp }}$.

The Jordan block of $\sigma$, denoted by $\operatorname{Jord}(\sigma)$, is a set of all pairs $(c, \rho)$ where $\rho \cong \widetilde{\rho}$ is an irreducible cuspidal representation of some $G L\left(n_{\rho}, F\right)$ and $c$ is a positive integer such that the following two conditions are satisfied:

1. $c$ is even if and only if $L(s, \rho, r)$ has a pole at $s=0$. The local $L$ function $L(s, \rho, r)$ is the one defined by Shahidi (see for instance [14], [15]), where $r=\bigwedge^{2} \mathbb{C}^{n_{\rho}}$ is the exterior-square representation of the standard representation on $\mathbb{C}^{n_{\rho}}$ of $G L\left(n_{\rho}, \mathbb{C}\right)$ if $G_{n}$ is a symplectic or even-orthogonal group, and $r=\mathrm{Sym}^{2} \mathbb{C}^{n_{\rho}}$ is the symmetric-square 
representation of the standard representation on $\mathbb{C}^{n_{\rho}}$ of $G L\left(n_{\rho}, \mathbb{C}\right)$ if $G_{n}$ is an odd-orthogonal group.

2. The induced representation $\delta\left(\left[\nu^{-(c-1) / 2} \rho, \nu^{(c-1) / 2} \rho\right]\right) \rtimes \sigma$ is irreducible.

To explain the notion of the $\epsilon$-function, we will first define Jordan triples. These are the triples of the form (Jord, $\sigma^{\prime}, \epsilon$ ) where

- $\sigma^{\prime}$ is an irreducible cuspidal representation of some $G_{n}$.

- Jord is a finite set (possibly empty) of pairs $(c, \rho)$, where $\rho$ is an irreducible selfcontragredient cuspidal representation of $G L\left(n_{\rho}, F\right)$ and $c$ is a positive integer which is even if and only if $L(s, \rho, r)$ has a pole at $s=0$ (for the local $L$-function as above). For an irreducible selfcontragredient cuspidal representation $\rho$ of $G L\left(n_{\rho}, F\right)$ we write $\operatorname{Jord}_{\rho}=\{c:(c, \rho) \in \operatorname{Jord}\}$. If $\operatorname{Jord}_{\rho} \neq \emptyset$ and $c \in \operatorname{Jord}_{\rho}$, we put $c_{-}=\max \left\{d \in \operatorname{Jord}_{\rho}: d<c\right\}$, if it exists.

- $\epsilon$ is a function defined on a subset of Jord $\cup($ Jord $\times$ Jord) and attains the values 1 and -1 . If $(c, \rho) \in$ Jord, then $\epsilon(c, \rho)$ is not defined if and only if $c$ is odd and $\left(c^{\prime}, \rho\right) \in \operatorname{Jord}\left(\sigma^{\prime}\right)$ for some positive integer $c^{\prime}$. Next, $\epsilon$ is defined on a pair $(c, \rho),\left(c^{\prime}, \rho^{\prime}\right) \in$ Jord if and only if $\rho \cong \rho^{\prime}$ and $c \neq c^{\prime}$.

The following compatibility conditions must hold for different $c, c^{\prime}, c^{\prime \prime} \in$ $\operatorname{Jord}_{\rho}$ :

1. If $\epsilon(c, \rho)$ is defined (hence $\epsilon\left(c^{\prime}, \rho\right)$ is also defined), then $\epsilon\left((c, \rho),\left(c^{\prime}, \rho\right)\right)=$ $\epsilon(c, \rho) \cdot \epsilon\left(c^{\prime}, \rho\right)^{-1}$.

2. $\epsilon\left((c, \rho),\left(c^{\prime \prime}, \rho\right)\right)=\epsilon\left((c, \rho),\left(c^{\prime}, \rho\right)\right) \cdot \epsilon\left(\left(c^{\prime}, \rho\right),\left(c^{\prime \prime}, \rho\right)\right)$.

3. $\epsilon\left((c, \rho),\left(c^{\prime}, \rho\right)\right)=\epsilon\left(\left(c^{\prime}, \rho\right),(c, \rho)\right)$.

Listed properties show that it is enough to know the value of $\epsilon$ on the consecutive pairs $\left(c_{-}, \rho\right),(c, \rho)$ and on the minimal element of $\operatorname{Jord}_{\rho}$ (if it is defined on elements, not only on pairs).

Suppose that, for the Jordan triple (Jord, $\left.\sigma^{\prime}, \epsilon\right)$, there is $(c, \rho) \in$ Jord such that $\epsilon\left(\left(c_{-}, \rho\right),(c, \rho)\right)=1$. If we put $\operatorname{Jord}^{\prime}=\operatorname{Jord} \backslash\left\{\left(c_{-}, \rho\right),(c, \rho)\right\}$ and consider the restriction $\epsilon^{\prime}$ of $\epsilon$ to $\operatorname{Jord}^{\prime} \cup\left(\operatorname{Jord}^{\prime} \times \operatorname{Jord}^{\prime}\right)$, we obtain a new Jordan triple $\left(\operatorname{Jord}^{\prime}, \sigma^{\prime}, \epsilon^{\prime}\right)$, and we say that such Jordan triple is subordinated to (Jord, $\left.\sigma^{\prime}, \epsilon\right)$.

We say that the Jordan triple (Jord, $\sigma^{\prime}, \epsilon$ ) is a triple of alternated type if we have $\epsilon\left(\left(c_{-}, \rho\right),(c, \rho)\right)=-1$ whenever $c_{-}$is defined and there is an increasing bijection $\phi_{\rho}: \operatorname{Jord}_{\rho} \rightarrow \operatorname{Jord}_{\rho}^{\prime}\left(\sigma^{\prime}\right)$, where $\operatorname{Jord}_{\rho}^{\prime}\left(\sigma^{\prime}\right)$ equals $\operatorname{Jord}_{\rho}\left(\sigma^{\prime}\right) \cup\{0\}$ if $a$ is even and $\epsilon\left(\min \operatorname{Jord}_{\rho}, \rho\right)=1$ and $\operatorname{Jord}_{\rho}^{\prime}\left(\sigma^{\prime}\right)$ equals $\operatorname{Jord}_{\rho}\left(\sigma^{\prime}\right)$ otherwise.

The Jordan triple (Jord, $\left.\sigma^{\prime}, \epsilon\right)$ dominates the Jordan triple ( $\operatorname{Jord}^{\prime}, \sigma^{\prime}, \epsilon^{\prime}$ ) if there is a sequence of Jordan triples $\left(\operatorname{Jord}_{i}, \sigma^{\prime}, \epsilon_{i}\right), 0 \leq i \leq k$, such that $\left(\operatorname{Jord}_{0}, \sigma^{\prime}, \epsilon_{0}\right)=\left(\operatorname{Jord}, \sigma^{\prime}, \epsilon\right),\left(\operatorname{Jord}_{k}, \sigma^{\prime}, \epsilon_{k}\right)=\left(\operatorname{Jord}^{\prime}, \sigma^{\prime}, \epsilon^{\prime}\right)$, and $\left(\operatorname{Jord}_{i}, \sigma^{\prime}, \epsilon_{i}\right)$ is subordinated to $\left(\operatorname{Jord}_{i-1}, \sigma^{\prime}, \epsilon_{i-1}\right)$ for $i \in\{1,2, \ldots, k\}$. The Jordan triple (Jord, $\sigma^{\prime}, \epsilon$ ) is called the admissible triple if it dominates a triple of alternated type. 
Classification given in [9] and [10] states that there is a one-to-one correspondence between the set of all discrete series in $\operatorname{Irr}(G)$ and the set of all admissible triples (Jord, $\sigma^{\prime}, \epsilon$ ) given by $\sigma=\sigma_{\left(\operatorname{Jord}, \sigma^{\prime}, \epsilon\right)}$, such that $\sigma_{\text {cusp }}=\sigma^{\prime}$ and $\operatorname{Jord}(\sigma)=$ Jord. Furthermore, if $(c, \rho) \in$ Jord is such that $\epsilon\left(\left(c_{-}, \rho\right),(c, \rho)\right)=1$, we set $\operatorname{Jord}^{\prime}=\operatorname{Jord} \backslash\left\{\left(c_{-}, \rho\right),(c, \rho)\right\}$ and consider the restriction $\epsilon^{\prime}$ of $\epsilon$ to $\operatorname{Jord}^{\prime} \cup\left(\operatorname{Jord}^{\prime} \times \operatorname{Jord}^{\prime}\right)$. Then $\left(\operatorname{Jord}^{\prime}, \sigma^{\prime}, \epsilon^{\prime}\right)$ is an admissible triple and $\sigma$ is a subrepresentation of

$$
\delta\left(\left[\nu^{-\left(c_{-}-1\right) / 2} \rho, \nu^{(c-1) / 2} \rho\right]\right) \rtimes \sigma_{\left(\operatorname{Jord}^{\prime}, \sigma^{\prime}, \epsilon^{\prime}\right)} .
$$

Such induced representation has exactly two discrete series subrepresentations, which are non-isomorphic. Moreover, the induced representation

$$
\delta\left(\left[\nu^{-\left(c_{-}-1\right) / 2} \rho, \nu^{\left(c_{-}-1\right) / 2} \rho\right]\right) \rtimes \sigma_{\left(\operatorname{Jord}^{\prime}, \sigma^{\prime}, \epsilon^{\prime}\right)}
$$

is the direct sum of two non-isomorphic tempered representations $\tau_{+}$and $\tau_{-}$ and there is the unique $\tau \in\left\{\tau_{+}, \tau_{-}\right\}$such that $\sigma$ is a subrepresentation of $\delta\left(\left[\nu^{\left(c_{-}+1\right) / 2} \rho, \nu^{(c-1) / 2} \rho\right]\right) \rtimes \tau$.

We shall also say that the discrete series $\sigma$ and its corresponding admissible triple (Jord, $\sigma^{\prime}, \epsilon$ ) are attached to each other.

It has been shown in [9, Proposition 5.3] and [10, Proposition 7.1] that triples of alternated type correspond to strongly positive discrete series. Thus, the strongly positive discrete series serve as the cornerstone in described construction of discrete series and an algebraic classification of such representations of metaplectic groups, which also holds in the case of classical groups, is given in [4]. Definition of the triples of alternated type implies that such discrete series are completely determined by their partial cuspidal support and the Jordan block. Since all discrete series which are studied in this paper share a common partial cuspidal support, it suffices to define only Jordan block when introducing some strongly positive discrete series. Such procedure has also been summarized in [11, Proposition 1.2].

We will also recall an inductive description of the non-supercuspidal strongly positive discrete series, obtained in [4, Theorem 5.1].

Proposition 2.2. Suppose that $\sigma_{s p} \in R(G)$ is an irreducible strongly positive representation and let $\rho \in \operatorname{Irr}(G L(m, F))$ denote an irreducible cuspidal representation such that some twist of $\rho$ appears in the cuspidal support of $\sigma_{s p}$. We denote by $\sigma_{\text {cusp }}$ the partial cuspidal support of $\sigma_{s p}$. Then there exist unique $a, b \in \mathbb{R}$ such that $a>0, b>0, b-a \in \mathbb{Z}_{\geq 0}$, and a unique irreducible strongly positive representation $\sigma_{s p}^{(1)}$ without $\nu^{a} \rho$ in the cuspidal support, with the property that $\sigma_{s p}$ is a unique irreducible subrepresentation of $\delta\left(\left[\nu^{a} \rho, \nu^{b} \rho\right]\right) \rtimes \sigma_{s p}^{(1)}$. Furthermore, there is a non-negative integer $l$ such that $s:=a+l>0$ and $\nu^{s} \rho \rtimes \sigma_{\text {cusp }}$ reduces. If $l=0$ there are no twists of $\rho$ appearing in the cuspidal support of $\sigma_{s p}^{(1)}$ and if $l>0$ there exist a unique 
$b^{\prime}>b$ and a unique strongly positive discrete series $\sigma_{s p}^{(2)}$, which contains neither $\nu^{a} \rho$ nor $\nu^{a+1} \rho$ in its cuspidal support, such that $\sigma_{s p}^{(1)}$ can be written as a unique irreducible subrepresentation of $\delta\left(\left[\nu^{a+1} \rho, \nu^{b^{\prime}} \rho\right]\right) \rtimes \sigma_{s p}^{(2)}$.

Here and subsequently we assume that $\sigma$ is a discrete series representation of $G_{n}$ and that there exist $c_{-}, c \in \operatorname{Jord}_{\rho^{\prime}}(\sigma)$ such that $\sigma$ is a subrepresentation of the induced representation of the form

$$
\delta\left(\left[\nu^{-\left(c_{-}-1\right) / 2} \rho^{\prime}, \nu^{(c-1) / 2} \rho^{\prime}\right]\right) \rtimes \sigma_{s p}
$$

for the strongly positive representation $\sigma_{s p}$ such that $\left[c_{-}, c\right] \cap \operatorname{Jord}_{\rho^{\prime}}\left(\sigma_{s p}\right)=\emptyset$. In other words, if we put $\operatorname{Jord}^{\prime}=\operatorname{Jord}(\sigma) \backslash\left\{\left(c_{-}, \rho^{\prime}\right),\left(c, \rho^{\prime}\right)\right\}$ and denote by $\epsilon^{\prime}$ the restriction of $\epsilon$ to $\mathrm{Jord}^{\prime}$, then $\sigma_{s p}$ corresponds to an admissible triple $\left(\mathrm{Jord}^{\prime}, \sigma_{\text {cusp }}, \epsilon^{\prime}\right)$ of alternated type.

Obviously, $\epsilon\left(\left(c_{-}, \rho\right),(c, \rho)\right)=1$ and pair $c_{-}, c$ with this property may not be unique. Since $\sigma_{s p}$ is strongly positive, there can be at most two such pairs.

We are interested in deriving necessary and sufficient conditions under which the generalized principal series $\delta \rtimes \sigma$, with $\delta=\delta\left(\left[\nu^{a} \rho, \nu^{b} \rho\right]\right), a>\frac{1}{2}$, and $\sigma$ as above, contains a discrete series subquotient. In particular, in this case the induced representation $\delta \rtimes \sigma$ reduces, so we may assume that $\operatorname{Jord}_{\rho} \neq \emptyset$ and that $a-c \in \mathbb{Z}, \forall 2 c+1 \in \operatorname{Jord}_{\rho}$ (this has already been observed in [11, Section 2] and also implies $\rho \cong \widetilde{\rho}$ ). Furthermore, by the last case considered in [3, Subsection 4.2], if $\delta \rtimes \sigma$ contains a discrete series subquotient then $2 a-1 \in \operatorname{Jord}_{\rho}$ and $2 b+1 \notin \operatorname{Jord}_{\rho}$.

To shorten the notation, we introduce the following concept:

DEFINITION 2.3. We call a generalized principal series $\delta \rtimes \sigma$ positive if the following conditions hold:

1. $\delta=\delta\left(\left[\nu^{a} \rho, \nu^{b} \rho\right]\right)$, where $a, b \in \mathbb{R}, b-a \in \mathbb{Z}_{\geq 0}, a>\frac{1}{2}$,

2. there exist $c_{-}, c \in \operatorname{Jord}_{\rho^{\prime}}(\sigma)$ such that $\sigma$ is a subrepresentation of the induced representation

$$
\delta\left(\left[\nu^{-\left(c_{-}-1\right) / 2} \rho^{\prime}, \nu^{(c-1) / 2} \rho^{\prime}\right]\right) \rtimes \sigma_{s p},
$$

for the strongly positive representation $\sigma_{s p}$ such that

$$
\left[c_{-}, c\right] \cap \operatorname{Jord}_{\rho^{\prime}}\left(\sigma_{s p}\right)=\emptyset,
$$

3. $\operatorname{Jord}_{\rho}(\sigma) \neq \emptyset$ and $a-c \in \mathbb{Z}, \forall 2 c+1 \in \operatorname{Jord}_{\rho}$,

4. $2 a-1 \in \operatorname{Jord}_{\rho}(\sigma)$ and $2 b+1 \notin \operatorname{Jord}_{\rho}(\sigma)$.

\section{Some technical Results}

This section is devoted to the proof of two technical results which will be frequently used later. 
LEMma 3.1. Let $\sigma \in \operatorname{Irr}\left(G_{n}\right)$ denote a discrete series representation and suppose that there there exist $c_{-}, c \in \operatorname{Jord}_{\rho^{\prime}}(\sigma)$ such that $\sigma$ is a subrepresentation of

$$
\delta\left(\left[\nu^{-\left(c_{-}-1\right) / 2} \rho^{\prime}, \nu^{(c-1) / 2} \rho^{\prime}\right]\right) \rtimes \sigma_{s p},
$$

for the strongly positive representation $\sigma_{s p}$ such that $\left[c_{-}, c\right] \cap \operatorname{Jord}_{\rho^{\prime}}\left(\sigma_{s p}\right)=\emptyset$. If $\sigma$ is written as a subrepresentation of the induced representation $\delta \rtimes \pi$, where $\delta$ is an irreducible essentially square-integrable representation of $G L\left(n_{\delta}, F\right)$, and $\pi$ a non-tempered representation of the form $L\left(\delta_{1} \times \delta_{2} \times \cdots \times \delta_{k} \rtimes \tau\right)$, then $k=1$.

Proof. We write $\delta=\delta\left(\left[\nu^{x} \rho, \nu^{y} \rho\right]\right)$ and $\pi=L\left(\delta_{1} \times \delta_{2} \times \cdots \times \delta_{k} \rtimes \tau\right)$ where $\delta_{i}=\delta\left(\left[\nu^{a_{i}} \rho_{i}, \nu^{b_{i}} \rho_{i}\right]\right)$ for $i=1,2, \ldots, k$, and $a_{1}+b_{1} \leq a_{2}+b_{2} \leq \cdots \leq$ $a_{k}+b_{k}<0$. To obtain a contradiction, suppose that $k \geq 2$.

Since $\pi$ is a subrepresentation of $\delta_{1} \rtimes L\left(\delta_{2} \times \cdots \times \delta_{k} \rtimes \tau\right)$, the squareintegrability of $\sigma$ shows that $\sigma$ is contained in the kernel of the intertwining operator

$$
\delta \times \delta_{1} \rtimes L\left(\delta_{2} \times \cdots \times \delta_{k} \rtimes \tau\right) \rightarrow \delta_{1} \times \delta \rtimes L\left(\delta_{2} \times \cdots \times \delta_{k} \rtimes \tau\right) .
$$

This also implies $\rho_{1} \cong \rho$. By [18], this kernel equals

$$
\delta\left(\left[\nu^{a_{1}} \rho, \nu^{y} \rho\right]\right) \times \delta\left(\left[\nu^{x} \rho, \nu^{b_{1}} \rho\right]\right) \rtimes L\left(\delta_{2} \times \cdots \times \delta_{k} \rtimes \tau\right) .
$$

Note that the representation $\delta\left(\left[\nu^{a_{1}} \rho, \nu^{y} \rho\right]\right) \times \delta\left(\left[\nu^{x} \rho, \nu^{b_{1}} \rho\right]\right)$ is irreducible. Furthermore, $L\left(\delta_{2} \times \cdots \times \delta_{k} \rtimes \tau\right)$ is a subrepresentation of $\delta_{2} \rtimes L\left(\delta_{3} \times \cdots \times \delta_{k} \rtimes \tau\right)$. This gives us an embedding

$$
\sigma \hookrightarrow \delta\left(\left[\nu^{a_{1}} \rho, \nu^{y} \rho\right]\right) \times \delta\left(\left[\nu^{x} \rho, \nu^{b_{1}} \rho\right]\right) \times \delta_{2} \rtimes L\left(\delta_{3} \times \cdots \times \delta_{k} \rtimes \tau\right) .
$$

Using Frobenius reciprocity, we obtain that the Jacquet module of $\sigma$ with respect to an appropriate parabolic subgroup contains

$$
\delta\left(\left[\nu^{a_{1}} \rho, \nu^{y} \rho\right]\right) \times \delta\left(\left[\nu^{x} \rho, \nu^{b_{1}} \rho\right]\right) \otimes \delta_{2} \otimes L\left(\delta_{3} \times \cdots \times \delta_{k} \rtimes \tau\right) .
$$

Since $\sigma$ is contained in $\delta\left(\left[\nu^{-\left(c_{-}-1\right) / 2} \rho^{\prime}, \nu^{(c-1) / 2} \rho^{\prime}\right]\right) \rtimes \sigma_{s p}$, applying Lemma 2.1 we obtain that there exist $i_{1}$ and $j_{1}$ such that $-\left(c_{-}+1\right) / 2 \leq i_{1} \leq j_{1} \leq$ $(c-1) / 2$ and $\pi_{1} \otimes \sigma_{1} \leq \mu^{*}\left(\sigma_{s p}\right)$ such that

$$
\begin{aligned}
\delta\left(\left[\nu^{a_{1}} \rho, \nu^{y} \rho\right]\right) \times \delta\left(\left[\nu^{x} \rho, \nu^{b_{1}} \rho\right]\right) \leq & \delta\left(\left[\nu^{-i_{1}} \rho^{\prime}, \nu^{\left(c_{-}-1\right) / 2} \rho^{\prime}\right]\right) \times \\
& \delta\left(\left[\nu^{j_{1}+1} \rho^{\prime}, \nu^{(c-1) / 2} \rho^{\prime}\right]\right) \times \pi_{1}
\end{aligned}
$$

and

$$
\delta_{2} \otimes L\left(\delta_{3} \times \cdots \times \delta_{k} \rtimes \tau\right) \leq \mu^{*}\left(\delta\left(\left[\nu^{i_{1}+1} \rho^{\prime}, \nu^{j_{1}} \rho^{\prime}\right]\right) \rtimes \sigma_{1}\right) .
$$

Since $\sigma_{s p}$ is strongly positive and $a_{1}<0,\left[6\right.$, Theorem 4.6] implies that $\rho \cong \rho^{\prime}$. Furthermore, either $-i_{1}=a_{1}$ or $j_{1}+1=a_{1}$ and, consequently, $i_{1} \cdot j_{1}>0$. 
Observe that $\sigma_{1}$ is also strongly positive so (3.1) gives $i_{1}<j_{1}$. We define $z=j_{1}$ if $i_{1}>0$ and $z=-i_{1}-1$ otherwise. It follows that

$$
\delta_{2} \otimes L\left(\delta_{3} \times \cdots \times \delta_{k} \rtimes \tau\right) \leq \mu^{*}\left(\delta\left(\left[\nu^{-a_{1}+1} \rho^{\prime}, \nu^{z} \rho^{\prime}\right]\right) \rtimes \sigma_{1}\right) .
$$

Here we have used the fact that for every irreducible essentially squareintegrable representation $\delta^{\prime}$ we have $\mu^{*}\left(\delta^{\prime} \rtimes \sigma_{1}\right)=\mu^{*}\left(\widetilde{\delta^{\prime}} \rtimes \sigma_{1}\right)$. Repeating the same procedure as before, we obtain that there exist $i_{2}$ and $j_{2}$ such that $-a_{1} \leq i_{2} \leq j_{2} \leq z$ and $\pi_{2} \otimes \sigma_{2} \leq \mu^{*}\left(\sigma_{1}\right)$ such that

$$
\delta\left(\left[\nu^{a_{2}} \rho_{2}, \nu^{b_{2}} \rho_{2}\right]\right) \leq \delta\left(\left[\nu^{-i_{2}} \rho^{\prime}, \nu^{a_{1}-1} \rho^{\prime}\right]\right) \times \delta\left(\left[\nu^{j_{2}+1} \rho^{\prime}, \nu^{z} \rho^{\prime}\right]\right) \times \pi_{2} .
$$

Now $a_{2}<0, j_{2}>0$ and strong positivity of $\sigma_{1}$ lead to $\rho_{2} \cong \rho^{\prime}, i_{2} \geq-a_{1}+1$, $a_{2}=-i_{2}$ and $b_{2}=a_{1}-1$. This contradicts our assumption $a_{1}+b_{1} \leq a_{2}+b_{2}$. Thus, $k=1$. This completes the proof.

In the last section we will also need the following correction of the part (iv) of [11, Theorem 4.1]. Let us shortly recall the required notation.

Let $\sigma_{s p}$ denote a strongly positive discrete series of $G_{n}$, attached to the admissible triple (Jord, $\sigma_{\text {cusp }}, \epsilon$ ) of alternated type. We suppose that $\delta$ is an irreducible essentially square-integrable representation of $G L\left(n_{\delta}, F\right)$ attached to the segment $\delta\left(\left[\nu^{-l_{1}} \rho, \nu^{l_{2}} \rho\right]\right)$ such that $l_{2}-l_{1} \in \mathbb{Z}_{>0}, l_{1}>0$ and $\operatorname{Jord}_{\rho} \neq \emptyset$, $l_{1}-c \in \mathbb{Z}, \forall 2 c+1 \in \operatorname{Jord}_{\rho}$. Furthermore, we assume that $\operatorname{Jord}_{\rho} \cap\left\{2 l_{1}+\right.$ $\left.1,2 l_{2}+1\right\}=\emptyset$ and that $\operatorname{Jord}_{\rho} \cap\left[2 l_{1}+1,2 l_{2}+1\right]$ is a singleton. Let us denote the unique element of the set $\operatorname{Jord}_{\rho} \cap\left[2 l_{1}+1,2 l_{2}+1\right]$ by $2 c+1$. Also, we introduce strongly positive discrete series $\sigma_{1}$ and $\sigma_{2}$ such that $\operatorname{Jord}\left(\sigma_{1}\right)=\operatorname{Jord} \backslash\{(2 c+$ $1, \rho)\} \cup\left\{\left(2 l_{1}+1, \rho\right)\right\}$ and $\operatorname{Jord}\left(\sigma_{2}\right)=\operatorname{Jord} \backslash\{(2 c+1, \rho)\} \cup\left\{\left(2 l_{2}+1, \rho\right)\right\}$. Finally, we define a discrete series $\sigma_{d s}$ whose corresponding admissible triple is $\left(\operatorname{Jord}^{\prime}, \sigma_{\text {cusp }}, \epsilon^{\prime}\right)$ such that $\operatorname{Jord}^{\prime}=\operatorname{Jord} \cup\left\{\left(2 l_{1}+1, \rho\right),\left(2 l_{2}+1, \rho\right)\right\}$ and $\epsilon^{\prime}\left((2 c+1, \rho),\left(2 l_{2}+1, \rho\right)\right)=\epsilon^{\prime}\left(\left(2 l_{1}+1, \rho\right),(2 c+1, \rho)\right)=1$, while on all other pairs $\epsilon^{\prime}$ equals -1 . The following result appears to be very important for our considerations.

Proposition 3.2. In $R(G)$ we have

$$
\delta \rtimes \sigma_{s p}=L(\widetilde{\delta} \rtimes \sigma)+L\left(\delta\left(\left[\nu^{-l_{2}} \rho, \nu^{c} \rho\right]\right) \rtimes \sigma_{1}\right)+L\left(\delta\left(\left[\nu^{-c} \rho, \nu^{l_{1}} \rho\right]\right) \rtimes \sigma_{2}\right)+\sigma_{d s} .
$$

Proof. It suffices to show that $\sigma_{d s}$ is the only tempered subquotient of $\delta \rtimes \sigma$. First we show that $\sigma_{d s}$ is a subrepresentation of $\delta \rtimes \sigma_{s p}$. Condition $\epsilon^{\prime}\left((2 c+1, \rho),\left(2 l_{2}+1, \rho\right)\right)=1$ gives the following embeddings:

$$
\sigma_{d s} \hookrightarrow \delta\left(\left[\nu^{-c} \rho, \nu^{l_{2}} \rho\right]\right) \rtimes \sigma_{1} \hookrightarrow \delta\left(\left[\nu^{-l_{1}} \rho, \nu^{l_{2}} \rho\right]\right) \times \delta\left(\left[\nu^{-c} \rho, \nu^{-l_{1}-1} \rho\right]\right) \rtimes \sigma_{1} .
$$

Using [10, Lemma 3.2], we deduce that there is some irreducible representation $\tau$ such that $\sigma_{d s}$ is a subrepresentation of $\delta\left(\left[\nu^{-l_{1}} \rho, \nu^{l_{2}} \rho\right]\right) \rtimes \tau$. Frobenius reciprocity gives $\mu^{*}\left(\sigma_{d s}\right) \geq \delta\left(\left[\nu^{-l_{1}} \rho, \nu^{l_{2}} \rho\right]\right) \otimes \tau$. We will show that the representation $\tau$ has to be a discrete series representation. Suppose, on the contrary, that $\tau$ is not a discrete series. Then [10, Section 8] shows that $\tau$ is a non-tempered representation and, using the previous lemma, we write $\tau$ as 
$L\left(\delta_{1} \rtimes \tau_{t}\right)$. Since $\delta_{1}=\delta\left(\left[\nu^{a_{1}} \rho_{1}, \nu^{b_{1}} \rho_{1}\right]\right)$ and $a_{1}+b_{1}<0$, from the cuspidal support of $\sigma_{d s}$ we obtain that $b_{1}<0$ and that $\rho_{1} \cong \rho$. In the same way as in the proof of the previous lemma we deduce that $\sigma_{d s}$ is contained in

$$
\delta\left(\left[\nu^{a_{1}} \rho, \nu^{l_{2}} \rho\right]\right) \times \delta\left(\left[\nu^{-l_{1}} \rho, \nu^{b_{1}} \rho\right]\right) \rtimes \tau_{t} .
$$

Since

$$
\delta\left(\left[\nu^{a_{1}} \rho, \nu^{l_{2}} \rho\right]\right) \times \delta\left(\left[\nu^{-l_{1}} \rho, \nu^{b_{1}} \rho\right]\right) \cong \delta\left(\left[\nu^{-l_{1}} \rho, \nu^{b_{1}} \rho\right]\right) \times \delta\left(\left[\nu^{a_{1}} \rho, \nu^{l_{2}} \rho\right]\right),
$$

we obtain that $b_{1}=-l_{1}-1$ and $\sigma_{d s} \hookrightarrow \delta\left(\left[\nu^{a_{1}} \rho, \nu^{l_{2}} \rho\right]\right) \rtimes \tau_{t}$. This also gives $l_{1}<-a_{1}<l_{2}$.

From $\sigma_{d s} \hookrightarrow \delta\left(\left[\nu^{-c} \rho, \nu^{l_{2}} \rho\right]\right) \rtimes \sigma_{1}$, applying Lemma 2.1 we obtain that there exist $i_{1}$ and $j_{1}$ such that $-c-1 \leq i_{1} \leq j_{1} \leq l_{2}$ and $\pi_{1} \otimes \sigma_{1}^{\prime} \leq \mu^{*}\left(\sigma_{1}\right)$ such that

$$
\delta\left(\left[\nu^{a_{1}} \rho, \nu^{l_{2}} \rho\right]\right) \leq \delta\left(\left[\nu^{-i_{1}} \rho, \nu^{c} \rho\right]\right) \times \delta\left(\left[\nu^{j_{1}+1} \rho, \nu^{l_{2}} \rho\right]\right) \times \pi_{1}
$$

and

$$
\tau_{t} \leq \mu^{*}\left(\delta\left(\left[\nu^{i_{1}+1} \rho, \nu^{j_{1}} \rho\right]\right) \rtimes \sigma_{1}^{\prime}\right) .
$$

Since $\sigma_{1}$ is strongly positive, [6, Theorem 4.6] implies $\left(i_{1}, j_{1}\right) \in\left\{\left(-c-1, a_{1}-\right.\right.$ $\left.1),\left(-a_{1}, c-1\right)\right\}$. This gives $\tau_{t} \leq \delta\left(\left[\nu^{-c} \rho, \nu^{a_{1}-1} \rho\right]\right) \rtimes \sigma_{1}$. From the cuspidal support of $\sigma_{d s}$ we obtain that $\tau_{t}$ is strongly positive, and [11, Proposition 3.1] implies $a_{1}=-c$. Thus, if $\tau$ is non-tempered then

$$
\tau \cong L\left(\delta\left(\left[\nu^{-c} \rho, \nu^{-l_{1}-1} \rho\right]\right) \rtimes \sigma_{1}\right) .
$$

On the other hand, condition $\epsilon^{\prime}\left(\left(2 l_{1}+1, \rho\right),(2 c+1, \rho)\right)=1$ implies $\mu^{*}\left(\sigma_{d s}\right) \geq \delta\left(\left[\nu^{l_{1}+1} \rho, \nu^{c} \rho\right]\right) \otimes \tau^{\prime}$ for some irreducible representation $\tau^{\prime}$. Since $\sigma_{d s}$ is a subrepresentation of $\delta\left(\left[\nu^{-l_{1}} \rho, \nu^{l_{2}} \rho\right]\right) \rtimes \tau$ and $l_{2}>c$, it is not hard to obtain $\mu^{*}(\tau) \geq \delta\left(\left[\nu^{l_{1}+1} \rho, \nu^{c} \rho\right]\right) \otimes \tau^{\prime \prime}$ for some irreducible representation $\tau^{\prime \prime}$.

By [11, Proposition 3.1], we have

$$
\delta\left(\left[\nu^{-c} \rho, \nu^{-l_{1}-1} \rho\right]\right) \rtimes \sigma_{1}=L\left(\delta\left(\left[\nu^{-c} \rho, \nu^{-l_{1}-1} \rho\right]\right) \rtimes \sigma_{1}\right)+\sigma_{s p} .
$$

Furthermore, [6, Theorem 4.6] implies that $\mu^{*}\left(\sigma_{s p}\right) \geq \delta\left(\left[\nu^{l_{1}+1} \rho, \nu^{c} \rho\right]\right) \otimes \sigma_{1}$. It follows directly from the definition of $\sigma_{1}$ and Lemma 2.1 that $\delta\left(\left[\nu^{l_{1}+1} \rho, \nu^{c} \rho\right]\right) \otimes$ $\sigma_{1}$ is the only irreducible constituent of the form $\delta\left(\left[\nu^{l_{1}+1} \rho, \nu^{c} \rho\right]\right) \otimes \tau^{\prime \prime}$ appearing in $\mu^{*}\left(\delta\left(\left[\nu^{-c} \rho, \nu^{-l_{1}-1} \rho\right]\right) \rtimes \sigma_{1}\right)$. Therefore, $\sigma_{d s}$ is not contained in $\delta \rtimes L\left(\delta\left(\left[\nu^{-c} \rho, \nu^{-l_{1}-1} \rho\right]\right) \rtimes \sigma_{1}\right)$.

This enables us to conclude that $\tau$ is a discrete series representation. Furthermore, from the cuspidal support of $\sigma_{d s}$ we see that $\tau$ is strongly positive. Using [6, Lemma 3.6] gives $\tau \cong \sigma_{s p}$, i.e., $\sigma_{d s} \hookrightarrow \delta \rtimes \sigma_{s p}$.

It remains to prove that $\sigma_{d s}$ is the only irreducible tempered subquotient of $\delta \rtimes \sigma_{s p}$.

Suppose, contrary to our claim, that there is some other tempered subquotient of $\delta \rtimes \sigma_{s p}$ and let us denote such subquotient by $\tau$. Using [10, Lemma 8.1], we deduce that $\tau$ is a discrete series. The description of cuspidal support of $\tau$ 
and Proposition 2.2 show that it is not a strongly positive representation. We assume that $\tau$ corresponds to the admissible triple ( $\left.\operatorname{Jord}^{(1)}, \sigma_{\text {cusp }}, \epsilon^{(1)}\right)$, where $\operatorname{Jord}^{(1)}=\operatorname{Jord} \cup\left\{\left(2 l_{1}+1, \rho\right),\left(2 l_{2}+1, \rho\right)\right\}$. Since $\tau$ is not strongly positive, there is some $\left(d, \rho^{\prime}\right) \in \operatorname{Jord}^{(1)}$ such that $\epsilon^{\prime}\left(\left(d_{-}, \rho^{\prime}\right),\left(d, \rho^{\prime}\right)\right)=1$. In the same way as in $\left[11\right.$, Lemma 4.8] we obtain that $\rho^{\prime} \cong \rho$ and that $d \in\left\{2 c+1,2 l_{2}+1\right\}$. If $d=2 c+1$, we obtain from [10] that $\mu^{*}(\tau) \geq \delta\left(\left[\nu^{-l_{1}} \rho, \nu^{c} \rho\right]\right) \otimes \sigma_{2}$, while if $d=2 l_{2}+1$ then $\mu^{*}(\tau) \geq \delta\left(\left[\nu^{-c} \rho, \nu^{l_{2}} \rho\right]\right) \otimes \sigma_{1}$. But, one readily sees that both these representation appear with multiplicity one in $\mu^{*}\left(\delta \rtimes \sigma_{s p}\right)$ and they also appear in $\mu^{*}\left(\sigma_{d s}\right)$. Consequently, such representations do not appear in $\mu^{*}(\tau)$.

This contradicts our assumption and proves the proposition.

\section{Discrete Series SUbquOtients}

In this section we determine conditions under which a discrete series representation appears in the composition series of the positive generalized discrete series $\delta \rtimes \sigma$, given as in Definition 2.3. Here and subsequently, we suppose that $\sigma$ is attached to the admissible triple (Jord, $\sigma_{c u s p}, \epsilon$ ), and we will use the same notation as in Definition 2.3.

Obviously, if $\sigma_{d s}$ is a discrete series subquotient of $\delta \rtimes \sigma$, then $\sigma_{d s}$ corresponds to the admissible triple $\left(\mathrm{Jord}^{(1)}, \sigma_{c u s p}, \epsilon^{(1)}\right)$, where $\mathrm{Jord}^{(1)}$ equals Jord $\backslash\{(\rho, 2 a-1)\} \cup\{(\rho, 2 b+1)\}$. Also, inspecting the cuspidal support of $\delta \rtimes \sigma$ and using the description of cuspidal support of a strongly positive discrete series given in [5, Theorem 2.1] and Proposition 2.2, we deduce that there is $d \in \operatorname{Jord}_{\rho^{\prime}}^{(1)}$ such that $\sigma_{d s}$ is a subrepresentation of

$$
\delta\left(\left[\nu^{-\left(d_{-}-1\right) / 2} \rho^{\prime}, \nu^{(d-1) / 2} \rho^{\prime}\right]\right) \rtimes \sigma_{s p}^{(1)},
$$

for the strongly positive discrete series $\sigma_{s p}^{(1)}$ such that $\operatorname{Jord}_{\rho^{\prime}}\left(\sigma_{s p}^{(1)}\right) \cap\left[d_{-}, d\right]=\emptyset$.

We start with the case which follows directly from [17, Theorem 8.2].

LEMMA 4.1. If $\operatorname{Jord}_{\rho} \cap[2 a+1,2 b+1]=\emptyset$, then the positive generalized principal series $\delta \rtimes \sigma$ contains a discrete series subquotient.

The rest of this section will be devoted to the case $\operatorname{Jord}_{\rho} \cap[2 a+1,2 b+1] \neq$ $\emptyset$. In the sequel, let $c_{\text {min }}$ denote the minimum of the set $\operatorname{Jord}_{\rho} \cap[2 a+1,2 b+1]$ and let $c_{\max }$ denote the maximum of the set $\operatorname{Jord}_{\rho} \cap[2 a+1,2 b+1]$. Note that in $\operatorname{Jord}_{\rho}$ we have $\left(c_{\text {min }}\right)_{-}=2 a-1$. First we have an elementary, but important, technical result.

LEMMA 4.2. If the positive generalized principal series $\delta \rtimes \sigma$ contains $a$ discrete series subquotient and $\operatorname{Jord}_{\rho} \cap[2 a+1,2 b+1] \neq \emptyset$, then $\epsilon((2 a-$ $\left.1, \rho),\left(c_{\min }, \rho\right)\right)=1$.

Proof. Suppose that $\sigma_{d s}$ is the discrete series subquotient of $\delta \rtimes \sigma$ which corresponds to the admissible triple $\left(\operatorname{Jord}^{(1)}, \sigma_{\text {cusp }}, \epsilon^{(1)}\right)$. Since $(\rho, 2 a-1) \notin$ 
$\operatorname{Jord}^{(1)},\left[17\right.$, Theorem 8.2] gives $\mu^{*}\left(\sigma_{d s}\right) \geq \delta\left(\left[\nu^{a} \rho, \nu^{\left(c_{\min }-1\right) / 2} \rho\right]\right) \otimes \pi$ for some irreducible representation $\pi$ of $G_{n^{\prime}}$. This also provides $\mu^{*}(\delta \rtimes \sigma) \geq$ $\delta\left(\left[\nu^{a} \rho, \nu^{\left(c_{\min }-1\right) / 2} \rho\right]\right) \otimes \pi$ and Lemma 2.1 implies that there exist $i$ and $j$ such that $a-1 \leq i \leq j \leq b$ and an irreducible constituent $\tau \otimes \sigma^{\prime}$ of $\mu^{*}(\sigma)$ such that

$$
\delta\left(\left[\nu^{a} \rho, \nu^{\left(c_{\min }-1\right) / 2} \rho\right]\right) \leq \delta\left(\left[\nu^{-i} \rho, \nu^{-a} \rho\right]\right) \times \delta\left(\left[\nu^{j+1} \rho, \nu^{b} \rho\right]\right) \times \tau .
$$

Since $a>0$ and $\left(c_{\min }-1\right) / 2$ is less than $b$, we deduce that $\mu^{*}(\sigma)$ contains $\delta\left(\left[\nu^{a} \rho, \nu^{\left(c_{\min }-1\right) / 2} \rho\right]\right) \otimes \pi^{\prime}$, for some irreducible representation $\pi^{\prime}$. Now [17, Proposition 7.2] implies $\epsilon\left((2 a-1, \rho),\left(c_{\min }, \rho\right)\right)=1$ and the lemma is proved.

In the following sequence of propositions, several possibilities will be studied separately.

Proposition 4.3. If $c_{\min }<\left(c_{\max }\right)_{\text {- then }}$ the positive generalized principal series does not contain a discrete series subquotient.

Proof. If $\epsilon\left((2 a-1, \rho),\left(c_{\min }, \rho\right)\right)=-1$, statement of the proposition follows from the previous lemma. Thus, in the rest of the proof we may assume that $\epsilon\left((2 a-1, \rho),\left(c_{\min }, \rho\right)\right)=1$. This also shows that $\rho \cong \rho^{\prime}$. We denote by $c$ an element of $\operatorname{Jord}_{\rho}$ such that $c_{\text {min }}=c_{-}$.

Two possibilities will be considered separately.

- $\left(c_{\max }\right)_{-} \neq c$.

Suppose, contrary to our assertion, that there exists a discrete series subquotient $\sigma_{d s}$ of $\delta \rtimes \sigma$, and that it corresponds to the admissible triple $\left(\operatorname{Jord}^{(1)}, \sigma_{\text {cusp }}, \epsilon^{(1)}\right)$. Then there exists $\left(d, \rho^{\prime \prime}\right) \in \operatorname{Jord}^{(1)}$ such that

$$
\epsilon^{(1)}\left(\left(d_{-}, \rho^{\prime \prime}\right),\left(d, \rho^{\prime \prime}\right)\right)=1 \text {. }
$$

This implies

$$
\mu^{*}\left(\sigma_{d s}\right) \geq \delta\left(\left[\nu^{-\left(d_{-}-1\right) / 2} \rho^{\prime \prime}, \nu^{(d-1) / 2} \rho^{\prime \prime}\right]\right) \otimes \sigma_{s p}^{(1)},
$$

for the appropriate strongly positive discrete series $\sigma_{s p}^{(1)}$. Since $\sigma_{d s}$ is an irreducible subquotient of $\delta \rtimes \sigma$, the same irreducible constituent appears in $\mu^{*}(\delta \rtimes \sigma)$. Using the structural formula, recalled in Lemma 2.1, we conclude that there exist $i$ and $j$ such that $a-1 \leq i \leq j \leq b$ and an irreducible constituent $\tau \otimes \sigma^{\prime}$ of $\mu^{*}(\sigma)$ such that

$$
\delta\left(\left[\nu^{-\left(d_{-}-1\right) / 2} \rho^{\prime \prime}, \nu^{(d-1) / 2} \rho^{\prime \prime}\right]\right) \leq \delta\left(\left[\nu^{-i} \rho, \nu^{-a} \rho\right]\right) \times \delta\left(\left[\nu^{j+1} \rho, \nu^{b} \rho\right]\right) \times \tau .
$$

Since by the definition of positive generalized principal series we have $a>\frac{1}{2}$, it follows that $\tau$ is of the form $\delta\left(\left[\nu^{k} \rho^{\prime \prime}, \nu^{l} \rho^{\prime \prime}\right]\right)$ for some $k<0$ and $l>0$. Now from the cuspidal support of $\sigma$ we deduce that $\rho^{\prime \prime} \cong \rho$.

If $(d-1) / 2=b$, then $d_{-}=c_{\max }$. Furthermore, since $2 b+1 \notin$ $\operatorname{Jord}_{\rho}$, we have either $i=\left(c_{\max }-1\right) / 2$ and $\tau \cong \delta\left(\left[\nu^{-a+1} \rho, \nu^{j} \rho\right]\right)$ or $\tau \cong$ $\delta\left(\left[\nu^{-\left(c_{\max }-1\right) / 2} \rho, \nu^{j} \rho\right]\right)$. These two cases will be studied separately. 
(i) Suppose $\tau \cong \delta\left(\left[\nu^{-a+1} \rho, \nu^{j} \rho\right]\right)$. Since $-a+1 \leq 0$ and $j \geq\left(c_{\max }+1\right) / 2$, it directly follows that $i=j$ and, using the transitivity of Jacquet modules, that

$$
\mu^{*}(\sigma) \geq \delta\left(\left[\nu^{\left(\left(c_{\max }\right)_{-}-1\right) / 2} \rho, \nu^{\left(c_{\max }-1\right) / 2} \rho\right]\right) \otimes \sigma^{\prime \prime},
$$

for some irreducible $\sigma^{\prime \prime}$. This gives $\epsilon\left(\left(\left(c_{\max }\right)_{-}, \rho\right),\left(c_{\max }, \rho\right)\right)=1$, which is impossible, since $\epsilon\left((2 a-1, \rho),\left(c_{\min }, \rho\right)\right)=1$ and the definition of positive generalized principal series implies $\epsilon\left(\left(x_{-}, \rho\right),(x, \rho)\right)=-1$ for $x>c$.

(ii) Suppose $\tau \cong \delta\left(\left[\nu^{-\left(c_{\max }-1\right) / 2} \rho, \nu^{j} \rho\right]\right)$. Using the well known fact that $\delta\left(\left[\nu^{-\left(c_{\max }-1\right) / 2} \rho, \nu^{j} \rho\right]\right) \otimes \sigma^{\prime} \leq \mu^{*}(\sigma)$ implies $2 j+1 \in \operatorname{Jord}_{\rho}$, we get a contradiction since the square-integrability of $\sigma$ implies $j>\left(c_{\max }-\right.$ 1) $/ 2$ and $j<b$.

Thus, $d \neq 2 b+1$ and it follows directly from the square-integrability of $\sigma$ that $\tau$ is of the form $\delta\left(\left[\nu^{k} \rho, \nu^{(d-1) / 2} \rho\right]\right)$, for some $k<0$. This implies $d \in \operatorname{Jord}_{\rho} \cap \operatorname{Jord}_{\rho}^{(1)}$ and, similarly as in the case $d=2 b+1$, we obtain that $\epsilon((x, \rho),(d, \rho))=1$, where $x$ is an element of $\operatorname{Jord}_{\rho}$ such that $\langle x, d\rangle \cap \operatorname{Jord}_{\rho}=\emptyset$. By Definition 2.3 and Lemma 4.2, either $d=c$ or $d=c_{\min }$. Note that $d=c$ implies $\epsilon\left(\left(c_{\min }, \rho\right),(c, \rho)\right)=1$, while $d=c_{\text {min }}$ implies $2 a-1>\min \left(\operatorname{Jord}_{\rho}\right)$. We will discuss only the case $d=c$, the other case can be handled in the same way but more easily.

Assumption $d=c$ implies $\epsilon^{(1)}\left(\left(c_{\min }, \rho\right),(c, \rho)\right)=1$. This clearly implies

$$
\mu^{*}(\delta \rtimes \sigma) \geq \delta\left(\left[\nu^{-\left(c_{\min }-1\right) / 2} \rho, \nu^{(c-1) / 2} \rho\right]\right) \otimes \sigma_{s p}^{\prime},
$$

where $\sigma_{s p}^{\prime}$ is the strongly positive discrete series such that $\operatorname{Jord}\left(\sigma_{s p}^{\prime}\right)=$ $\operatorname{Jord}(\sigma) \backslash\left\{\left(c_{\min }, \rho\right),(c, \rho)\right\}$. Using Lemma 2.1, we obtain that there exist $i$ and $j$ such that $a-1 \leq i \leq j \leq b$ and $\pi \otimes \tau^{\prime} \leq \mu^{*}(\sigma)$ such that

$$
\delta\left(\left[\nu^{-\left(c_{\min }-1\right) / 2} \rho, \nu^{(c-1) / 2} \rho\right]\right) \leq \delta\left(\left[\nu^{-i} \rho, \nu^{-a} \rho\right]\right) \times \delta\left(\left[\nu^{j+1} \rho, \nu^{b} \rho\right]\right) \times \pi
$$

and $\sigma_{s p}^{\prime} \leq \delta\left(\left[\nu^{i+1} \rho, \nu^{j} \rho\right]\right) \rtimes \tau^{\prime}$. Since $b>(c-1) / 2$, it follows that $j=b$. By the square-integrability of $\sigma$, we have the following two possibilities:

(a) $i=a-1$. This gives $\pi \cong \delta\left(\left[\nu^{-\left(c_{\min }-1\right) / 2} \rho, \nu^{(c-1) / 2} \rho\right]\right)$ and $[12$, Theorem 2.3] shows that $\tau^{\prime}$ is the strongly positive discrete series such that $\operatorname{Jord}\left(\tau^{\prime}\right)=\operatorname{Jord}(\sigma) \backslash\left\{\left(c_{\text {min }}, \rho\right),(c, \rho)\right\}$. It follows that $\sigma_{s p}^{\prime}$ is an irreducible subquotient of the induced representation $\delta\left(\left[\nu^{a} \rho, \nu^{b} \rho\right]\right) \rtimes \tau^{\prime}$, contradicting [11, Proposition 3.1].

(b) $i=\left(c_{\min }-1\right) / 2$. This gives $\pi \cong \delta\left(\left[\nu^{-a+1} \rho, \nu^{(c-1) / 2} \rho\right]\right)$. Using the embedding

$$
\sigma \hookrightarrow \delta\left(\left[\nu^{-\left(c_{\min }-1\right) / 2} \rho, \nu^{(c-1) / 2} \rho\right]\right) \rtimes \sigma_{s p},
$$

where $\sigma_{s p}$ is the strongly positive discrete series such that $\operatorname{Jord}\left(\sigma_{s p}\right)=$ $\operatorname{Jord}(\sigma) \backslash\left\{\left(c_{\min }, \rho\right),(c, \rho)\right\}$, it directly follows that $\tau^{\prime}$ is an irreducible 
subquotient of $\delta\left(\left[\nu^{a} \rho, \nu^{\left(c_{\min }-1\right) / 2} \rho\right]\right) \rtimes \sigma_{s p}$. This gives

$$
\sigma_{s p}^{\prime} \leq \delta\left(\left[\nu^{\left(c_{\min }+1\right) / 2} \rho, \nu^{b} \rho\right]\right) \times \delta\left(\left[\nu^{a} \rho, \nu^{\left(c_{\min }-1\right) / 2} \rho\right]\right) \rtimes \sigma_{s p} .
$$

It follows from [6, Theorem 4.6] that $\mu^{*}\left(\sigma_{s p}^{\prime}\right) \geq \delta\left(\left[\nu^{a} \rho, \nu^{(x-1) / 2} \rho\right]\right) \otimes$ $\sigma_{s p}^{\prime \prime}$, for $x \in \operatorname{Jord}_{\rho}$ such that $x_{-}=c$ and strongly positive discrete series $\sigma_{s p}^{\prime \prime}$ such that $\operatorname{Jord}\left(\sigma_{s p}^{\prime \prime}\right)=\operatorname{Jord}\left(\sigma_{s p}^{\prime}\right) \backslash\{(x, \rho)\} \cup\{(2 a-1, \rho)\}$. Applying the structural formula for $\mu^{*}$ on the right-hand side of (4.1), we obtain that there exist $i_{1}, i_{2}, j_{1}, j_{2}$ such that $\left(c_{\min }-1\right) / 2 \leq i_{1} \leq j_{1} \leq b$, $a-1 \leq i_{2} \leq j_{2} \leq\left(c_{\min }-1\right) / 2$ and $\pi^{\prime} \otimes \sigma^{\prime} \leq \mu^{*}\left(\sigma_{s p}\right)$ such that

$$
\begin{aligned}
\delta\left(\left[\nu^{a} \rho, \nu^{(x-1) / 2} \rho\right]\right) \leq & \delta\left(\left[\nu^{-i_{1}} \rho, \nu^{-\left(c_{\min }+1\right) / 2} \rho\right]\right) \times \delta\left(\left[\nu^{j_{1}+1} \rho, \nu^{b} \rho\right]\right) \times \\
& \delta\left(\left[\nu^{-i_{2}} \rho, \nu^{-a} \rho\right]\right) \times \delta\left(\left[\nu^{j_{2}+1} \rho, \nu^{\left(c_{\min }-1\right) / 2} \rho\right]\right) \times \pi^{\prime}
\end{aligned}
$$

and

$$
\sigma_{s p}^{\prime \prime} \leq \delta\left(\left[\nu^{i_{1}+1} \rho, \nu^{j_{1}} \rho\right]\right) \times \delta\left(\left[\nu^{i_{2}+1} \rho, \nu^{j_{2}} \rho\right]\right) \rtimes \sigma^{\prime} .
$$

Since $a>0, b>(x-1) / 2$ and $c_{\min }>0$, we deduce that $i_{1}=\left(c_{\min }-\right.$ 1) $/ 2, j_{1}=b$ and $i_{2}=a-1$. This gives

$$
\delta\left(\left[\nu^{a} \rho, \nu^{(x-1) / 2} \rho\right]\right) \leq \delta\left(\left[\nu^{j_{2}+1} \rho, \nu^{\left(c_{\min }-1\right) / 2} \rho\right]\right) \times \pi^{\prime} .
$$

Applying [6, Theorem 4.6], we directly obtain that $j_{2}=a-1$ and $\pi^{\prime} \cong \delta\left(\left[\nu^{\left(c_{\min }+1\right) / 2} \rho, \nu^{(x-1) / 2} \rho\right]\right)$. Using [6, Theorem 4.6] again, we get that $\sigma^{\prime}$ is the strongly positive discrete series such that $\operatorname{Jord}\left(\sigma^{\prime}\right)=$ $\operatorname{Jord}\left(\sigma_{s p}\right) \backslash\{(x, \rho)\} \cup\left\{\left(c_{\min }, \rho\right)\right\}$. This implies $\sigma_{s p}^{\prime \prime} \leq \delta\left(\left[\nu^{a} \rho, \nu^{b} \rho\right]\right) \rtimes \sigma^{\prime}$, contradicting [11, Proposition 3.1].

This ends the proof in the case $\left(c_{\max }\right)_{-} \neq c$. We will now consider the remaining case.

- $\left(c_{\max }\right)_{-}=c$.

Again we suppose, contrary to our claim, that there is a discrete series subquotient $\sigma_{d s}$ of $\delta \rtimes \sigma$. We denote by $\left(\operatorname{Jord}^{(1)}, \sigma_{\text {cusp }}, \epsilon^{(1)}\right)$ the admissible triple attached to $\sigma_{d s}$. Again, there must be some $\left(d, \rho^{\prime \prime}\right) \in \operatorname{Jord}^{(1)}$ such that $\epsilon^{(1)}\left(\left(d_{-}, \rho^{\prime \prime}\right),\left(d, \rho^{\prime \prime}\right)\right)=1$. In the same way as in the case $\left(c_{\max }\right)_{-} \neq c$ we conclude that $\rho \cong \rho^{\prime \prime}$ and $d$ equals either $c$ or $c_{\min }$. First, if we assume that $2 a-1 \neq \min \left(\operatorname{Jord}_{\rho}\right)$, then $d=c_{\text {min }}$ leads to a contradiction in the same way as in the previous case. Furthermore, if $2 a-1 \neq \min \left(\operatorname{Jord}_{\rho}\right)$ and $d=c$, then the properties of $\epsilon$-functions summarized in Section 2 imply that we have either $\epsilon^{(1)}\left(\left(\left(c_{\min }\right)_{-}, \rho\right),\left(c_{\min }, \rho\right)\right)=1$ or $\epsilon^{(1)}((c, \rho),(x, \rho))=1$, for $x \in \operatorname{Jord}_{\rho}$ such that $x_{-}=c$. We have already seen that both these situations are impossible.

It remains to consider the case $d=c$ and $2 a-1=\min \left(\operatorname{Jord}_{\rho}\right)$. Obviously, we can assume that $\epsilon^{(1)}\left(\left(\left(c_{\min }\right), \rho\right),(c, \rho)\right)=1$ and that $\epsilon^{(1)}((c, \rho),(x, \rho))=-1$ for an $x \in \operatorname{Jord}_{\rho}$ such that $x_{-}=c$. Using [17, Theorem 8.2] we deduce that $\sigma_{d s} \hookrightarrow \delta\left(\left[\nu^{a} \rho, \nu^{\left(c_{\min }-1\right) / 2} \rho\right]\right) \rtimes \sigma_{d s}^{\prime}$, for discrete series $\sigma_{d s}^{\prime}$ attached to the 
admissible triple $\left(\operatorname{Jord}^{(2)}, \sigma_{\text {cusp }}, \epsilon^{(2)}\right)$ such that $\operatorname{Jord}^{(2)}=\operatorname{Jord}^{(1)} \backslash\left\{\left(c_{\min }, \rho\right)\right\} \cup$ $\{(2 a-1, \rho)\}$ and $\epsilon^{(2)}((2 a-1, \rho),(c, \rho))=1$. Furthermore, from [10, Section 9] and $[11$, Theorem 1.1] we get

$$
\sigma_{d s}^{\prime} \hookrightarrow \delta\left(\left[\nu^{a} \rho, \nu^{(c-1) / 2} \rho\right]\right) \rtimes \tau
$$

for the tempered subrepresentation $\tau$ of $\delta\left(\left[\nu^{-a+1} \rho, \nu^{a-1} \rho\right]\right) \rtimes \sigma_{s p}^{\prime}$, where $\sigma_{s p}^{\prime}$ is the strongly positive discrete series such that $\operatorname{Jord}\left(\sigma_{s p}^{\prime}\right)=\operatorname{Jord}^{(2)} \backslash\{(2 a-$ $1, \rho),(c, \rho)\}$. This gives

$$
\sigma_{d s} \hookrightarrow \delta\left(\left[\nu^{a} \rho, \nu^{\left(c_{\min }-1\right) / 2} \rho\right]\right) \times \delta\left(\left[\nu^{a} \rho, \nu^{(c-1) / 2} \rho\right]\right) \rtimes \tau .
$$

Since the induced representation $\delta\left(\left[\nu^{a} \rho, \nu^{\left(c_{\min }-1\right) / 2} \rho\right]\right) \times \delta\left(\left[\nu^{a} \rho, \nu^{(c-1) / 2} \rho\right]\right)$ is irreducible, Frobenius reciprocity gives

$$
\mu^{*}\left(\sigma_{d s}\right) \geq \delta\left(\left[\nu^{a} \rho, \nu^{\left(c_{\min }-1\right) / 2} \rho\right]\right) \times \delta\left(\left[\nu^{a} \rho, \nu^{(c-1) / 2} \rho\right]\right) \otimes \tau .
$$

Thus, the same irreducible representation has to be contained in $\mu^{*}(\delta \rtimes \sigma)$. Since $\sigma$ is a subrepresentation of the induced representation of the form $\delta\left(\left[\nu^{-\left(c_{\min }-1\right) / 2} \rho, \nu^{(c-1) / 2} \rho\right]\right) \rtimes \sigma_{s p}$, where $\sigma_{s p}$ denotes the strongly positive discrete series such that $\operatorname{Jord}\left(\sigma_{s p}\right)=\operatorname{Jord} \backslash\left\{\left(c_{\min }, \rho\right),(c, \rho)\right\}$, we deduce that there exist $i_{1}, i_{2}, j_{1}, j_{2}$ such that $a-1 \leq i_{1} \leq j_{1} \leq b,-\left(c_{\min }-1\right) / 2-1 \leq$ $i_{2} \leq j_{2} \leq(c-1) / 2$ and $\pi \otimes \sigma^{\prime} \leq \mu^{*}\left(\sigma_{s p}\right)$ such that

$$
\begin{aligned}
& \delta\left(\left[\nu^{a} \rho, \nu^{\left(c_{\min }-1\right) / 2} \rho\right]\right) \times \delta\left(\left[\nu^{a} \rho, \nu^{(c-1) / 2} \rho\right]\right) \\
& \leq \delta\left(\left[\nu^{-i_{1}} \rho, \nu^{-a} \rho\right]\right) \times \delta\left(\left[\nu^{j_{1}+1} \rho, \nu^{b} \rho\right]\right) \\
& \quad \times \delta\left(\left[\nu^{-i_{2}} \rho, \nu^{\left(c_{\min }-1\right) / 2} \rho\right]\right) \times \delta\left(\left[\nu^{j_{2}+1} \rho, \nu^{(c-1) / 2} \rho\right]\right) \times \pi
\end{aligned}
$$

and

$$
\tau \leq \delta\left(\left[\nu^{i_{1}+1} \rho, \nu^{j_{1}} \rho\right]\right) \times \delta\left(\left[\nu^{i_{2}+1} \rho, \nu^{j_{2}} \rho\right]\right) \rtimes \sigma^{\prime} .
$$

In analogous way as before, using $a>0,(c-1) / 2<b$ and [6, Theorem 4.6], we deduce that $i_{1}=a-1, j_{1}=b, i_{2}=a$ and $j_{2}=a-1$. Now we can rewrite $(4.2)$ as

$$
\tau \leq \delta\left(\left[\nu^{a} \rho, \nu^{b} \rho\right]\right) \times \delta\left(\left[\nu^{-a+1} \rho, \nu^{a-1} \rho\right]\right) \rtimes \sigma_{s p} .
$$

We have already observed that $\mu^{*}(\tau)$ contains $\delta\left(\left[\nu^{-a+1} \rho, \nu^{a-1} \rho\right]\right) \otimes \sigma_{s p}^{\prime}$. Applying the structural formula to the right-hand side of (4.3), we conclude that $\sigma_{s p}^{\prime}$ is an irreducible subquotient of $\delta\left(\left[\nu^{a} \rho, \nu^{b} \rho\right]\right) \rtimes \sigma_{s p}$. This contradicts [11, Proposition 3.1], since for $\left(c_{\max }, \rho\right) \in \operatorname{Jord}\left(\sigma_{s p}\right)$ we have $2 a-1<c_{\max }<2 b+1$. This completes the proof.

Proposition 4.4. If $\epsilon\left((2 a-1, \rho),\left(c_{\min }, \rho\right)\right)=1$ and $c_{\min }=c_{\max }$, then the positive generalized principal series contains a discrete series subquotient. 
Proof. To shorten the notation, we write $c=c_{\text {min }}$. Let $\sigma_{s p}$ denote the strongly positive discrete series such that $\operatorname{Jord}\left(\sigma_{s p}\right)=\operatorname{Jord}(\sigma) \backslash\{(2 a-$ $1, \rho),(c, \rho)\}$. Then $\sigma$ is a subrepresentation of $\delta\left(\left[\nu^{-a+1} \rho, \nu^{(c-1) / 2} \rho\right]\right) \rtimes \sigma_{s p}$. By the classification of discrete series, this induced representation has one more discrete series subrepresentation, which we denote by $\sigma^{\prime}$. Similarly, the induced representation $\delta\left(\left[\nu^{-(c-1) / 2} \rho, \nu^{b} \rho\right]\right) \rtimes \sigma_{s p}$ contains two discrete series subrepresentations, let us denote them by $\sigma_{d s}^{(1)}$ and $\sigma_{d s}^{(2)}$. For $i \in\{1,2\}$, we suppose that $\sigma_{d s}^{(i)}$ is attached to the admissible triple $\left(\operatorname{Jord}^{(i)}, \sigma_{c u s p}, \epsilon^{(i)}\right)$. Note that $\epsilon^{(i)}((c, \rho),(2 b+1, \rho))=1$ and we have the following embeddings:

$$
\begin{aligned}
\sigma_{d s}^{(i)} & \hookrightarrow \delta\left(\left[\nu^{-(c-1) / 2} \rho, \nu^{b} \rho\right]\right) \rtimes \sigma_{s p} \\
& \hookrightarrow \delta\left(\left[\nu^{a} \rho, \nu^{b} \rho\right]\right) \times \delta\left(\left[\nu^{-(c-1) / 2} \rho, \nu^{a-1} \rho\right]\right) \rtimes \sigma_{s p} .
\end{aligned}
$$

By [11, Theorem 2.1], in $R(G)$ we have

$$
\delta\left(\left[\nu^{-(c-1) / 2} \rho, \nu^{a-1} \rho\right]\right) \rtimes \sigma_{s p}=\sigma+\sigma^{\prime}+L\left(\delta\left(\left[\nu^{-a+1} \rho, \nu^{(c-1) / 2} \rho\right]\right) \rtimes \sigma_{s p}\right) .
$$

Let us prove that $\sigma_{d s}^{(i)}, i \in\{1,2\}$, is not a subquotient of the induced representation

$$
\delta\left(\left[\nu^{a} \rho, \nu^{b} \rho\right]\right) \rtimes L\left(\delta\left(\left[\nu^{-a+1} \rho, \nu^{(c-1) / 2} \rho\right]\right) \rtimes \sigma_{s p}\right) .
$$

It follows from [17, Theorem 8.2] that $\mu^{*}\left(\sigma_{d s}^{(i)}\right) \geq \delta\left(\left[\nu^{a} \rho, \nu^{(c-1) / 2} \rho\right]\right) \otimes \tau$, for some irreducible representation $\tau$. Assume, on the contrary, that $\sigma_{d s}^{(i)}$ is a subquotient of $\delta\left(\left[\nu^{a} \rho, \nu^{b} \rho\right]\right) \rtimes L\left(\delta\left(\left[\nu^{-a+1} \rho, \nu^{(c-1) / 2} \rho\right]\right) \rtimes \sigma_{s p}\right)$. The irreducible representation $\delta\left(\left[\nu^{a} \rho, \nu^{(c-1) / 2} \rho\right]\right) \otimes \tau$ is then contained in the Jacquet module of $\delta\left(\left[\nu^{a} \rho, \nu^{b} \rho\right]\right) \rtimes L\left(\delta\left(\left[\nu^{-a+1} \rho, \nu^{(c-1) / 2} \rho\right]\right) \rtimes \sigma_{s p}\right)$ with respect to the appropriate maximal parabolic subgroup. Applying Lemma 2.1, we deduce that there exist $j_{1}$ and $j_{2}$ such that $a-1 \leq j_{1} \leq j_{2} \leq b$ and an irreducible constituent $\pi \otimes \tau^{\prime}$ of $\mu^{*}\left(L\left(\delta\left(\left[\nu^{-a+1} \rho, \nu^{(c-1) / 2} \rho\right]\right) \rtimes \sigma_{s p}\right)\right)$ such that

$$
\delta\left(\left[\nu^{a} \rho, \nu^{(c-1) / 2} \rho\right]\right) \leq \delta\left(\left[\nu^{-j_{1}} \rho, \nu^{-a} \rho\right]\right) \times \delta\left(\left[\nu^{j_{2}+1} \rho, \nu^{b} \rho\right]\right) \times \pi .
$$

Using $a>0$ and $(c-1) / 2<b$, we obtain that

$$
\mu^{*}\left(L\left(\delta\left(\left[\nu^{-a+1} \rho, \nu^{(c-1) / 2} \rho\right]\right) \rtimes \sigma_{s p}\right)\right) \geq \delta\left(\left[\nu^{a} \rho, \nu^{(c-1) / 2} \rho\right]\right) \otimes \tau^{\prime} .
$$

It follows from [10, Corollary 4.7] that exactly two irreducible constituents of the form $\delta\left(\left[\nu^{a} \rho, \nu^{(c-1) / 2} \rho\right]\right) \otimes \tau^{\prime}$ appear in $\mu^{*}\left(\delta\left(\left[\nu^{-(c-1) / 2} \rho, \nu^{a-1} \rho\right]\right) \rtimes \sigma_{s p}\right)$ and, by [10, Section 9] and [11, Theorem 2.1], none of them appears in $\mu^{*}\left(L\left(\delta\left(\left[\nu^{-a+1} \rho, \nu^{(c-1) / 2} \rho\right]\right) \rtimes \sigma_{s p}\right)\right)$, a contradiction.

Thus, $\sigma_{d s}^{(i)}$ is contained in

$$
\delta\left(\left[\nu^{a} \rho, \nu^{b} \rho\right]\right) \rtimes \sigma+\delta\left(\left[\nu^{a} \rho, \nu^{b} \rho\right]\right) \rtimes \sigma^{\prime},
$$

for $i \in\{1,2\}$. It remains to prove that neither of the positive generalized principal series $\delta \rtimes \sigma$ and $\delta \rtimes \sigma^{\prime}$ can contain both representations $\sigma_{d s}^{(1)}$ and $\sigma_{d s}^{(2)}$. Note that $\mu^{*}\left(\sigma_{d s}^{(i)}\right) \geq \delta\left(\left[\nu^{-(c-1) / 2} \rho, \nu^{b} \rho\right]\right) \otimes \sigma_{s p}$ for $i \in\{1,2\}$. Now we 
calculate the multiplicity of $\delta\left(\left[\nu^{-(c-1) / 2} \rho, \nu^{b} \rho\right]\right) \otimes \sigma_{s p}$ in $\mu^{*}(\delta \rtimes \sigma)$. There exist $i$ and $j$ such that $a-1 \leq i \leq j \leq b$ and $\pi \otimes \sigma^{\prime \prime} \leq \mu^{*}(\sigma)$ such that

$$
\delta\left(\left[\nu^{-(c-1) / 2} \rho, \nu^{b} \rho\right]\right) \leq \delta\left(\left[\nu^{-i} \rho, \nu^{-a} \rho\right]\right) \times \delta\left(\left[\nu^{j+1} \rho, \nu^{b} \rho\right]\right) \times \pi
$$

and

$$
\sigma_{s p} \leq \delta\left(\left[\nu^{i+1} \rho, \nu^{j} \rho\right]\right) \rtimes \sigma^{\prime \prime}
$$

Since $2 b+1 \notin \operatorname{Jord}_{\rho}(\sigma)$ and $c=c_{\text {max }}$, it follows that $j \leq(c-1) / 2$. Also, using the square-integrability of $\sigma$ we deduce that $i=(c-1) / 2$. Hence $i=j$ and $\pi \cong \delta\left(\left[\nu^{-a+1} \rho, \nu^{(c-1) / 2} \rho\right]\right)$. It directly follows that $\sigma^{\prime \prime} \cong \sigma_{s p}$ and, since the representation $\delta\left(\left[\nu^{-a+1} \rho, \nu^{(c-1) / 2} \rho\right]\right) \otimes \sigma_{s p}$ appears in $\mu^{*}(\sigma)$ with multiplicity one, we get that the multiplicity of $\delta\left(\left[\nu^{-(c-1) / 2} \rho, \nu^{b} \rho\right]\right) \otimes \sigma_{s p}$ in $\mu^{*}(\delta \rtimes \sigma)$ also equals one.

In analogous way we deduce that the multiplicity of $\delta\left(\left[\nu^{-(c-1) / 2} \rho, \nu^{b} \rho\right]\right) \otimes$ $\sigma_{s p}$ in $\mu^{*}\left(\delta \rtimes \sigma^{\prime}\right)$ also equals one. Therefore, neither of representations $\delta \rtimes \sigma$ and $\delta \rtimes \sigma^{\prime}$ can contain both representations $\sigma_{d s}^{(1)}$ and $\sigma_{d s}^{(2)}$, and it follows that each contains exactly one of them. This completes the proof.

Proposition 4.5. If $\epsilon\left((2 a-1, \rho),\left(c_{\min }, \rho\right)\right)=1$ and $c_{\min }=\left(c_{\max }\right)_{-}$, then the positive generalized principal series contains a discrete series subquotient if and only if $\epsilon\left(\left(c_{\min }, \rho\right),\left(c_{\max }, \rho\right)\right)=1$.

Proof. As in the proof of the previous proposition, we denote by $\sigma_{s p}$ the strongly positive discrete series such that $\operatorname{Jord}\left(\sigma_{s p}\right)=\operatorname{Jord}(\sigma) \backslash\{(2 a-$ $\left.1, \rho),\left(c_{\min }, \rho\right)\right\}$ and by $\sigma^{\prime}$ the discrete series subrepresentation of the induced representation $\delta\left(\left[\nu^{-a+1} \rho, \nu^{\left(c_{\min }-1\right) / 2} \rho\right]\right) \rtimes \sigma_{s p}$ different than $\sigma$. Suppose that $\sigma$ is attached to the admissible triple (Jord, $\left.\sigma_{c u s p}, \epsilon\right)$ with $\epsilon\left(\left(c_{\min }, \rho\right),\left(c_{\max }, \rho\right)\right)=$ 1. Also, let $\sigma^{\prime}$ correspond to the admissible triple (Jord, $\left.\sigma_{\text {cusp }}, \epsilon^{\prime}\right)$. Then we have $\epsilon^{\prime}\left(\left(c_{\min }, \rho\right),\left(c_{\max }, \rho\right)\right)=-1$ and $\epsilon(((2 a-1)-\rho),(2 a-1, \rho))=1$, if $2 a-1 \neq \min \left(\operatorname{Jord}_{\rho}\right)$. Furthermore, let us denote by $\sigma_{s p}^{\prime}$ the strongly positive discrete series such that $\sigma$ is a subrepresentation of

$$
\delta\left(\left[\nu^{-\left(c_{\min }-1\right) / 2} \rho, \nu^{\left(c_{\max }-1\right) / 2} \rho\right]\right) \rtimes \sigma_{s p}^{\prime}
$$

and by $\sigma_{s p}^{\prime \prime}$ the strongly positive discrete series such that $\operatorname{Jord}\left(\sigma_{s p}^{\prime \prime}\right)=$ $\operatorname{Jord}\left(\sigma_{s p}^{\prime}\right) \backslash\{(2 a-1, \rho)\} \cup\{(2 b+1, \rho)\}$. Then the induced representation $\delta\left(\left[\nu^{-\left(c_{\min }-1\right) / 2} \rho, \nu^{\left(c_{\max }-1\right) / 2} \rho\right]\right) \rtimes \sigma_{s p}^{\prime \prime}$ has two irreducible subrepresentation which are both in discrete series. We denote these two representations by $\sigma_{d s}^{(1)}$ and $\sigma_{d s}^{(2)}$ and observe the following embeddings and intertwining operator, for 
$i \in\{1,2\}:$

$$
\begin{aligned}
\sigma_{d s}^{(i)} & \hookrightarrow \delta\left(\left[\nu^{-\left(c_{\min }-1\right) / 2} \rho, \nu^{\left(c_{\max }-1\right) / 2} \rho\right]\right) \rtimes \sigma_{s p}^{\prime \prime} \\
& \hookrightarrow \delta\left(\left[\nu^{-\left(c_{\min }-1\right) / 2} \rho, \nu^{\left(c_{\max }-1\right) / 2} \rho\right]\right) \times \delta\left(\left[\nu^{a} \rho, \nu^{b} \rho\right]\right) \rtimes \sigma_{s p}^{\prime} \\
& \rightarrow \delta\left(\left[\nu^{a} \rho, \nu^{b} \rho\right]\right) \times \delta\left(\left[\nu^{-\left(c_{\min }-1\right) / 2} \rho, \nu^{\left(c_{\max }-1\right) / 2} \rho\right]\right) \rtimes \sigma_{s p}^{\prime} .
\end{aligned}
$$

Let $\sigma^{\prime \prime}$ be the discrete series subrepresentation of the induced representation $\delta\left(\left[\nu^{-\left(c_{\min }-1\right) / 2} \rho, \nu^{\left(c_{\max }-1\right) / 2} \rho\right]\right) \rtimes \sigma_{s p}^{\prime}$, different than $\sigma$. If $\sigma^{\prime \prime}$ is attached to the admissible triple (Jord, $\left.\sigma_{\text {cusp }}, \epsilon^{\prime \prime}\right)$, then $\epsilon^{\prime \prime}\left((2 a-1, \rho),\left(c_{\min }, \rho\right)\right)=-1$. In $R(G)$ we have

$$
\begin{aligned}
& \delta\left(\left[\nu^{-\left(c_{\min }-1\right) / 2} \rho, \nu^{\left(c_{\max }-1\right) / 2} \rho\right]\right) \rtimes \sigma_{s p}^{\prime} \\
& \quad=\sigma+\sigma^{\prime \prime}+L\left(\delta\left(\left[\nu^{-\left(c_{\max }-1\right) / 2} \rho, \nu^{\left(c_{\min }-1\right) / 2} \rho\right]\right) \rtimes \sigma_{s p}^{\prime}\right),
\end{aligned}
$$

and one can see, in the same way as in the proof of the previous proposition, that $\sigma_{d s}^{(i)}$ is not an irreducible subquotient of

$$
\delta \rtimes L\left(\delta\left(\left[\nu^{-\left(c_{\min }-1\right) / 2} \rho, \nu^{\left(c_{\max }-1\right) / 2} \rho\right]\right) \rtimes \sigma_{s p}^{\prime}\right) .
$$

Also, [17, Theorem 8.2] shows that $\mu^{*}\left(\sigma_{d s}^{(i)}\right), i \in\{1,2\}$, contains an irreducible constituent of the form $\delta\left(\left[\nu^{a} \rho, \nu^{\left(c_{\min }-1\right) / 2} \rho\right]\right) \otimes \tau$. Thus, if for some irreducible representation $\tau^{\prime}$ the induced representation $\delta \rtimes \tau^{\prime}$ contains $\sigma_{d s}^{(i)}$, for some $i \in\{1,2\}$, Lemma 2.1 and the fact that $\left(c_{\min }-1\right) / 2$ is less than $b$ imply $\mu^{*}\left(\tau^{\prime}\right) \geq \delta\left(\left[\nu^{a} \rho, \nu^{\left(c_{\min }-1\right) / 2} \rho\right]\right) \otimes \tau^{\prime \prime}$ for some irreducible representation $\tau^{\prime \prime}$. Now [17, Proposition 7.2] shows that $\sigma_{d s}^{(i)}, i \in\{1,2\}$, is not an irreducible subquotient of $\delta \rtimes \sigma^{\prime \prime}$ and, consequently, both representations $\sigma_{d s}^{(1)}$ and $\sigma_{d s}^{(2)}$ appear in the composition series of $\delta \rtimes \sigma$.

It remains to prove that the positive generalized principal series $\delta \rtimes \sigma^{\prime}$ does not contain a discrete series subquotient.

Let as first assume that discrete series $\sigma_{d s}^{(1)}$ is attached to the admissible triple $\left(\operatorname{Jord}^{(1)}, \sigma_{\text {cusp }}, \epsilon^{(1)}\right)$ such that $2 a-1 \neq \min \left(\operatorname{Jord}_{\rho}\right)$ and $\epsilon^{(1)}\left(\left((2 a-1)_{-}, \rho\right),\left(c_{\min }, \rho\right)\right)=1$. To simplify the notation, we denote $(2 a-1)_{-}$by $x$. From [10, Section 9] and [11, Theorem 1.1] then follows that $\mu^{*}\left(\sigma_{d s}^{(1)}\right) \geq \delta\left(\left[\nu^{(x+1) / 2} \rho, \nu^{\left(c_{\min }-1\right) / 2} \rho\right]\right) \otimes \tau_{1}$, where $\tau_{1}$ is one of the tempered subrepresentations of $\delta\left(\left[\nu^{-(x-1) / 2} \rho, \nu^{(x-1) / 2} \rho\right]\right) \rtimes \sigma_{s p}^{(1)}$, for the strongly positive discrete series $\sigma_{s p}^{(1)}$ such that $\operatorname{Jord}\left(\sigma_{s p}^{(1)}\right)=\operatorname{Jord}\left(\sigma_{s p}^{\prime \prime}\right) \backslash\{(x, \rho)\} \cup\left\{\left(c_{\max }, \rho\right)\right\}$. Since $\sigma_{d s}^{(1)}$ is a subquotient of $\delta \rtimes \sigma$, it follows that

$$
\mu^{*}\left(\delta \times \delta\left(\left[\nu^{-a+1} \rho, \nu^{\left(c_{\min }-1\right) / 2} \rho\right]\right) \rtimes \sigma_{s p}\right) \geq \delta\left(\left[\nu^{(x+1) / 2} \rho, \nu^{\left(c_{\min }-1\right) / 2} \rho\right]\right) \otimes \tau_{1} .
$$

Using Lemma 2.1, in analogous way as before we deduce that $\tau_{1}$ is an irreducible tempered subquotient of

$$
\delta\left(\left[\nu^{a} \rho, \nu^{b} \rho\right]\right) \times \delta\left(\left[\nu^{-(x-1) / 2} \rho, \nu^{a-1} \rho\right]\right) \rtimes \sigma_{s p} .
$$


Now we calculate the multiplicity of $\delta\left(\left[\nu^{-(x-1) / 2} \rho, \nu^{(x-1) / 2} \rho\right]\right) \otimes \sigma_{s p}^{(1)}$ in (4.4). There exist $i_{1}, i_{2}, j_{1}, j_{2}$ such that $a-1 \leq i_{1} \leq j_{1} \leq b,-(x-1) / 2-1 \leq i_{2} \leq$ $j_{2} \leq a-1$ and $\pi \otimes \sigma^{\prime \prime} \leq \mu^{*}\left(\sigma_{s p}\right)$ such that

$$
\begin{aligned}
\delta\left(\left[\nu^{-(x-1) / 2} \rho, \nu^{(x-1) / 2} \rho\right]\right) \leq & \delta\left(\left[\nu^{-i_{1}} \rho, \nu^{-a} \rho\right]\right) \times \delta\left(\left[\nu^{j_{1}+1} \rho, \nu^{b} \rho\right]\right) \times \\
& \delta\left(\left[\nu^{-i_{2}} \rho, \nu^{(x-1) / 2} \rho\right]\right) \times \delta\left(\left[\nu^{j_{2}+1} \rho, \nu^{a-1} \rho\right]\right) \times \pi
\end{aligned}
$$

and

$$
\sigma_{s p}^{(1)} \leq \delta\left(\left[\nu^{i_{1}+1} \rho, \nu^{j_{1}} \rho\right]\right) \times \delta\left(\left[\nu^{i_{2}+1} \rho, \nu^{j_{2}} \rho\right]\right) \rtimes \sigma^{\prime \prime} .
$$

Since $(x-1) / 2<a-1, a-1<b$ and $\sigma_{s p}$ is strongly positive, it follows $i_{1}=a-1, j_{1}=b, j_{2}=a-1$ and $i_{2}=(x-1) / 2$. This gives

$$
\sigma_{s p}^{(1)} \leq \delta\left(\left[\nu^{a} \rho, \nu^{b} \rho\right]\right) \times \delta\left(\left[\nu^{(x+1) / 2} \rho, \nu^{a-1} \rho\right]\right) \rtimes \sigma_{s p} .
$$

By $\left[6\right.$, Theorem 4.6], $\mu^{*}\left(\sigma_{s p}^{(1)}\right)$ contains $\delta\left(\left[\nu^{(x+1) / 2} \rho, \nu^{\left(c_{\max }-1\right) / 2} \rho\right]\right) \otimes \sigma_{s p}^{\prime \prime}$. Inspecting $\mu^{*}$ of the right-hand side of (4.5), since no representation of the form $\delta\left(\left[\nu^{(x+1) / 2} \rho, \nu^{\left(c_{\max }-1\right) / 2} \rho\right]\right) \otimes \sigma^{\prime \prime \prime}$ appears in $\mu^{*}\left(\sigma_{s p}\right)$, we obtain that $\sigma_{s p}^{\prime \prime}$ is contained in $\delta\left(\left[\nu^{a} \rho, \nu^{b} \rho\right]\right) \rtimes \sigma_{s p}^{(2)}$, where $\sigma_{s p}^{(2)}$ is the strongly positive discrete series such that $\operatorname{Jord}\left(\sigma_{s p}^{(2)}\right)=\operatorname{Jord}\left(\sigma_{s p}\right) \backslash\left\{\left(c_{\max }, \rho\right)\right\} \cup\{(2 a-3, \rho)\}$. From $[11$, Proposition 3.1] follows that it is contained there with multiplicity one.

Therefore, if we denote by $\tau_{2}$ the tempered subrepresentation of the induced representation $\delta\left(\left[\nu^{-(x-1) / 2} \rho, \nu^{(x-1) / 2} \rho\right]\right) \rtimes \sigma_{s p}^{(1)}$ different than $\tau_{1}$, then $\tau_{2}$ is not an irreducible subquotient of (4.4). We recall that $\tau_{1}$ and $\tau_{2}$ are not isomorphic.

We also note that $\sigma_{s p}^{(1)}$ is an irreducible subquotient of

$$
\delta\left(\left[\nu^{a} \rho, \nu^{b} \rho\right]\right) \rtimes L\left(\delta\left(\left[\nu^{(x+1) / 2} \rho, \nu^{a-1} \rho\right]\right) \rtimes \sigma_{s p}\right),
$$

so this induced representation reduces.

Suppose that there is some discrete series subquotient $\sigma_{d s}^{(3)}$ of $\delta \rtimes \sigma^{\prime}$ and denote the admissible triple attached to $\sigma_{d s}^{(3)}$ by $\left(\operatorname{Jord}^{(1)}, \sigma_{c u s p}, \epsilon^{(3)}\right)$. Then there is $\left(d, \rho^{\prime \prime}\right) \in \operatorname{Jord}^{(1)}$ such that $\epsilon^{(3)}\left(\left(d_{-}, \rho^{\prime \prime}\right),\left(d, \rho^{\prime \prime}\right)\right)=1$. In the same way as in the proof of Proposition 4.3 we deduce that $\rho^{\prime \prime} \cong \rho$ and $d \in\left\{c_{\min }, c_{\max }, 2 b+1\right\}$. Since we have already seen that $\sigma_{d s}^{(3)} ¥ \sigma_{d s}^{(1)}$ and $\sigma_{d s}^{(3)} \approx \sigma_{d s}^{(2)}$, we obtain that $\epsilon^{(3)}\left(\left(c_{\max }, \rho\right),(2 b+1, \rho)\right)=\epsilon^{(3)}\left(\left(c_{\min }, \rho\right),\left(c_{\max }, \rho\right)\right)=-1$. Properties of the $\epsilon$-function imply $d=c_{\text {min }}$ and $x=\min \left(\operatorname{Jord}_{\rho}\right)$, for $x$ as before. Since $\mu^{*}\left(\sigma_{d s}^{(3)}\right) \geq \delta\left(\left[\nu^{(x+1) / 2} \rho, \nu^{\left(c_{\min }-1\right) / 2} \rho\right]\right) \otimes \tau_{2}$ and $\sigma^{\prime}$ is a subrepresentation of $\delta\left(\left[\nu^{-a+1} \rho, \nu^{\left(c_{\min }-1\right) / 2} \rho\right]\right) \rtimes \sigma_{s p}$, it follows that

$$
\mu^{*}\left(\delta \times \delta\left(\left[\nu^{-a+1} \rho, \nu^{\left(c_{\min }-1\right) / 2} \rho\right]\right) \rtimes \sigma_{s p}\right)
$$

also contains $\delta\left(\left[\nu^{(x+1) / 2} \rho, \nu^{\left(c_{\min }-1\right) / 2} \rho\right]\right) \otimes \tau_{2}$. It is not hard to see that this can happen only if $\tau_{2}$ is an irreducible subquotient of the induced representation 
given in (4.4), and we have already seen that this is impossible. Thus, the proposition is proved.

In the following theorem we summarize the main results obtained in this section.

THEOREM 4.6. The positive generalized principal series $\delta \rtimes \sigma$ contains a discrete series subquotient if and only if one of the following holds:

(i) $\operatorname{Jord}_{\rho} \cap[2 a+1,2 b+1]=\emptyset$.

(ii) $\operatorname{Jord}_{\rho} \cap[2 a+1,2 b+1] \neq \emptyset$ and, for $c_{\min }$ and $c_{\max }$ such that $c_{\min }=$ $\min \left(\operatorname{Jord}_{\rho} \cap[2 a+1,2 b+1]\right)$ and $c_{\max }=\max \left(\operatorname{Jord}_{\rho} \cap[2 a+1,2 b+1]\right)$, we have $\epsilon\left((2 a-1, \rho),\left(c_{\min }, \rho\right)\right)=1$ and either

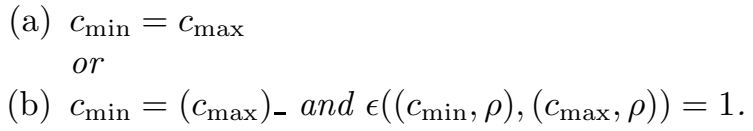

\section{Discrete SERIES SUbrepresentations}

In this section we identify a discrete series subrepresentation for every positive generalized principal series which contains a discrete series subquotient.

We start with the most elementary case, which follows directly from $[17$, Theorem 8.2].

Proposition 5.1. If $\operatorname{Jord}_{\rho} \cap[2 a+1,2 b+1]=\emptyset$, then the positive generalized principal series $\delta \rtimes \sigma$ contains a discrete series subrepresentation.

In the rest of this section we are concerned with the case $\operatorname{Jord}_{\rho} \cap[2 a+$ $1,2 b+1] \neq \emptyset$. As in the previous section, we denote by $c_{\min }$ the minimum of the set $\operatorname{Jord}_{\rho} \cap[2 a+1,2 b+1]$ and by $c_{\max }$ the maximum of the same set. We suppose that $\delta \rtimes \sigma$ has a discrete series subquotient and write $\sigma$ as an irreducible subrepresentation of $\delta\left(\left[\nu^{-a+1} \rho, \nu^{\left(c_{\min }-1\right) / 2} \rho\right]\right) \rtimes \sigma_{s p}$, where $\sigma_{s p}$ is the strongly positive discrete series such that $\operatorname{Jord}_{\rho}\left(\sigma_{s p}\right) \cap\left[2 a-1, c_{\text {min }}\right]=\emptyset$.

Proposition 5.2. Suppose that $\epsilon\left((2 a-1, \rho),\left(c_{\min }, \rho\right)\right)=1$ and $c_{\min }=$ $c_{\max }$. Then the positive generalized principal series contains a discrete series subrepresentation.

Proof. As in the proof of Proposition 4.4, we write $c=c_{\min }$ and denote by $\sigma_{d s}$ a discrete series subrepresentation of $\delta\left(\left[\nu^{-(c-1) / 2} \rho, \nu^{b} \rho\right]\right) \rtimes \sigma_{s p}$ which is contained in $\delta \rtimes \sigma$.

Obviously, we have $\sigma_{d s} \hookrightarrow \delta \times \delta\left(\left[\nu^{-(c-1) / 2} \rho, \nu^{a-1} \rho\right]\right) \rtimes \sigma_{s p}$ and, by [10, Lemma 3.2] there is some irreducible representation $\pi$ such that $\sigma_{d s}$ is a subrepresentation of $\delta\left(\left[\nu^{a} \rho, \nu^{b} \rho\right]\right) \rtimes \pi$. We will show that $\pi \cong \sigma$. 
Let us first show that the representation $\pi$ has to be a discrete series representation. Suppose, on the contrary, that $\pi$ is not a discrete series representation. Then, from the cuspidal support of $\pi$, we deduce that it is a nontempered representation. Using Lemma 3.1, we write $\pi$ in the form $L\left(\delta_{1} \rtimes \tau\right)$, with $\delta_{1}=\delta\left(\left[\nu^{a_{1}} \rho_{1}, \nu^{b_{1}} \rho_{1}\right]\right), a_{1}+b_{1}<0$.

This implies

$$
\sigma_{d s} \hookrightarrow \delta \times \delta_{1} \rtimes \tau \rightarrow \delta_{1} \times \delta \rtimes \tau .
$$

The square-integrability of $\sigma_{d s}$ shows that this representation is contained in the kernel of the last intertwining operator. Thus, $\rho_{1} \cong \rho$ and $a-1 \leq b_{1} \leq$ $b-1$. It follows that $\sigma_{d s}$ is a subrepresentation of

$$
\delta\left(\left[\nu^{a_{1}} \rho, \nu^{b} \rho\right]\right) \times \delta\left(\left[\nu^{a} \rho, \nu^{b_{1}} \rho\right]\right) \rtimes \tau,
$$

which is isomorphic to

$$
\delta\left(\left[\nu^{a} \rho, \nu^{b_{1}} \rho\right]\right) \times \delta\left(\left[\nu^{a_{1}} \rho, \nu^{b} \rho\right]\right) \rtimes \tau .
$$

There are two possibilities to consider:

- $b_{1}>a-1$.

Now (5.2) implies that $\nu^{b_{1}} \rho \otimes \tau^{\prime}$ appears in $\mu^{*}\left(\sigma_{d s}\right)$ for some irreducible representation $\tau^{\prime}$ and, by [10, Lemma 3.6], $\left(2 b_{1}+1, \rho\right) \in \operatorname{Jord}\left(\sigma_{d s}\right)$. Since $a_{1}+b_{1}<0$ and, by (5.1), $a_{1}+b>0$, we deduce that $2 b_{1}+1=c$, i.e., $b_{1}=(c-1) / 2$. Using (5.1) again we see that $\mu^{*}\left(\sigma_{d s}\right) \geq \delta\left(\left[\nu^{a_{1}} \rho, \nu^{b} \rho\right]\right) \otimes \tau^{\prime \prime}$ for some irreducible representation $\tau^{\prime \prime}$ and $a_{1}<-(c-1) / 2$, which is impossible by the definition of $\sigma_{d s}$.

- $b_{1}=a-1$.

Using Frobenius reciprocity, from (5.1) we obtain that

$$
\mu^{*}\left(\sigma_{d s}\right) \geq \delta\left(\left[\nu^{a_{1}} \rho, \nu^{b} \rho\right]\right) \otimes \tau .
$$

Since $\sigma_{d s}$ is contained in $\delta\left(\left[\nu^{-(c-1) / 2} \rho, \nu^{b} \rho\right]\right) \rtimes \sigma_{s p}$, using Lemma 2.1 we get that there exist $i$ and $j$ such that $-(c+1) / 2 \leq i \leq j \leq b$ and $\pi^{\prime} \otimes \sigma^{\prime} \leq \mu^{*}\left(\sigma_{s p}\right)$ such that

$$
\delta\left(\left[\nu^{a_{1}} \rho, \nu^{b} \rho\right]\right) \leq \delta\left(\left[\nu^{-i} \rho, \nu^{(c-1) / 2} \rho\right]\right) \times \delta\left(\left[\nu^{j+1} \rho, \nu^{b} \rho\right]\right) \times \pi^{\prime}
$$

and

$$
\tau \leq \delta\left(\left[\nu^{i} \rho, \nu^{j+1} \rho\right]\right) \rtimes \sigma^{\prime} .
$$

In the same way as before, we obtain that either $i=-a_{1}$ or $j+1=a_{1}$. Furthermore, it follows from [6, Theorem 4.6] that if $\nu^{x} \rho \otimes \tau^{\prime}$ appears in $\mu^{*}\left(\sigma_{s p}\right)$ then $x<(c-1) / 2$ or $b<x$. It follows that $\tau$ is a subquotient of $\delta\left(\left[\nu^{-a_{1}+1} \rho, \nu^{(c-1) / 2} \rho\right]\right) \rtimes \sigma_{s p}$. Since $\tau$ has to be a tempered representation, we conclude that $a_{1}=-(c-1) / 2$ and $\tau \cong \sigma_{s p}$. Consequently, $\pi \cong L\left(\delta\left(\left[\nu^{-(c-1) / 2} \rho, \nu^{a-1} \rho\right]\right) \rtimes \sigma_{s p}\right)$.

On the other hand, using [17, Theorem 8.2] we deduce that $\mu^{*}\left(\sigma_{d s}\right) \geq$ $\delta\left(\left[\nu^{a} \rho, \nu^{(c-1) / 2} \rho\right]\right) \otimes \sigma_{d s}^{\prime}$ for an appropriate discrete series $\sigma_{d s}^{\prime}$. Consequently, 
$\mu^{*}(\delta \rtimes \pi) \geq \delta\left(\left[\nu^{a} \rho, \nu^{(c-1) / 2} \rho\right]\right) \otimes \sigma_{d s}^{\prime}$ and $(c-1) / 2<b$ implies that $\mu^{*}(\pi) \geq$ $\delta\left(\left[\nu^{a} \rho, \nu^{(c-1) / 2} \rho\right]\right) \otimes \pi^{\prime}$, for some irreducible representation $\pi^{\prime}$. Representation $L\left(\delta\left(\left[\nu^{-(c-1) / 2} \rho, \nu^{a-1} \rho\right]\right) \rtimes \sigma_{s p}\right)$ is an irreducible quotient of the induced representation $\delta\left(\left[\nu^{-a+1} \rho, \nu^{(c-1) / 2} \rho\right]\right) \rtimes \sigma_{s p}$. It follows from [10, Corollary 4.7] that $\mu^{*}\left(\delta\left(\left[\nu^{-a+1} \rho, \nu^{(c-1) / 2} \rho\right]\right) \rtimes \sigma_{s p}\right)$ contains exactly two irreducible constituents of the form $\delta\left(\left[\nu^{a} \rho, \nu^{(c-1) / 2} \rho\right]\right) \otimes \pi^{\prime}$, which both appear in the Jacquet modules of discrete series subrepresentations of $\delta\left(\left[\nu^{-a+1} \rho, \nu^{(c-1) / 2} \rho\right]\right) \rtimes \sigma_{s p}$. Therefore, $\pi \approx L\left(\delta\left(\left[\nu^{-(c-1) / 2} \rho, \nu^{a-1} \rho\right]\right) \rtimes \sigma_{s p}\right)$.

Consequently, $\pi$ is a discrete series representation and we denote by $\left(\operatorname{Jord}^{\prime}, \sigma_{\text {cusp }}, \epsilon^{\prime}\right)$ the corresponding admissible triple. It directly follows that Jord $^{\prime}=$ Jord. We have already seen that $\mu^{*}(\pi) \geq \delta\left(\left[\nu^{a} \rho, \nu^{(c-1) / 2} \rho\right]\right) \otimes \pi^{\prime}$, which implies $\epsilon^{\prime}((2 a-1, \rho),(c, \rho))=1$, and $\pi$ is a subrepresentation of $\delta\left(\left[\nu^{-a+1} \rho, \nu^{(c-1) / 2} \rho\right]\right) \rtimes \sigma_{s p}$. If $\pi \not \sigma$ then it can be concluded in the same way as in the proof of Proposition 4.4 that $\sigma_{d s}$ is not an irreducible subquotient of $\delta \rtimes \pi$. Therefore, $\pi \cong \sigma$ and the proof is complete.

The remaining case is treated in the following proposition.

Proposition 5.3. Suppose that $\epsilon\left((2 a-1, \rho),\left(c_{\min }, \rho\right)\right)=1, c_{\min }=$ $\left(c_{\max }\right)_{\text {_ and }} \epsilon\left(\left(c_{\min }, \rho\right),\left(c_{\max }, \rho\right)\right)=1$. Then the positive generalized principal series contains a discrete series subrepresentation.

Proof. Let us denote by $\sigma_{s p}^{\prime}$ the strongly positive discrete series such that $\operatorname{Jord}\left(\sigma_{s p}^{\prime}\right)=\operatorname{Jord}\left(\sigma_{s p}\right) \backslash\left\{\left(c_{\text {max }}, \rho\right)\right\} \cup\{(2 b+1, \rho)\}$ and by $\sigma_{s p}^{\prime \prime}$ the strongly positive discrete series such that $\operatorname{Jord}\left(\sigma_{s p}^{\prime \prime}\right)=\operatorname{Jord}\left(\sigma_{s p}\right) \backslash\left\{\left(c_{\max }, \rho\right)\right\} \cup$ $\left\{\left(c_{\min }, \rho\right)\right\}$. Then the induced representations

$$
\delta\left(\left[\nu^{-\left(c_{\min }-1\right) / 2} \rho, \nu^{\left(c_{\max }-1\right) / 2} \rho\right]\right) \rtimes \sigma_{s p}^{\prime}
$$

and

$$
\delta\left(\left[\nu^{-\left(c_{\max }-1\right) / 2} \rho, \nu^{b} \rho\right]\right) \rtimes \sigma_{s p}^{\prime \prime}
$$

have a unique common irreducible subrepresentation, which is a discrete series representation and we denote it by $\sigma_{d s}$. Also, we denote the admissible triple attached to $\sigma_{d s}$ by $\left(\operatorname{Jord}^{\prime}, \sigma_{c u s p}, \epsilon^{\prime}\right)$.

From (5.4) follows that there is some irreducible representation $\pi$ such that $\sigma_{d s}$ is a subrepresentation of $\delta \rtimes \pi$. We will show that $\pi$ is a discrete series representation. Let us first show some results regarding the Jacquet modules of $\pi$, which will be used later.

Using the Frobenius reciprocity, from (5.3) we deduce that $\mu^{*}\left(\sigma_{d s}\right) \geq$ $\delta\left(\left[\nu^{-\left(c_{\min }-1\right) / 2} \rho, \nu^{\left(c_{\max }-1\right) / 2} \rho\right]\right) \otimes \sigma_{s p}^{\prime}$. Since $\sigma_{d s}$ is a subrepresentation of $\delta \rtimes \pi$, using Lemma 2.1, we deduce that there exist $i$ and $j$ such that $a-1 \leq i \leq j \leq b$ and $\delta^{\prime} \otimes \pi^{\prime} \leq \mu^{*}(\pi)$ such that

$$
\delta\left(\left[\nu^{-\left(c_{\min }-1\right) / 2} \rho, \nu^{\left(c_{\max }-1\right) / 2} \rho\right]\right) \leq \delta\left(\left[\nu^{-i} \rho, \nu^{-a} \rho\right]\right) \times \delta\left(\left[\nu^{j+1} \rho, \nu^{b} \rho\right]\right) \times \delta^{\prime} .
$$


From $b>\left(c_{\max }-1\right) / 2$ follows that $j=b$, so $\delta^{\prime}$ has to be equal either $\delta\left(\left[\nu^{-\left(c_{\min }-1\right) / 2} \rho, \nu^{\left(c_{\max }-1\right) / 2} \rho\right]\right)$ or $\delta\left(\left[\nu^{-a+1} \rho, \nu^{\left(c_{\max }-1\right) / 2} \rho\right]\right)$. In any case, using the transitivity of Jacquet modules, we obtain that

$$
\mu^{*}(\pi) \geq \delta\left(\left[\nu^{-a+1} \rho, \nu^{\left(c_{\max }-1\right) / 2} \rho\right]\right) \otimes \pi^{\prime}
$$

for some irreducible representation $\pi^{\prime}$. This also allows us to conclude that

$$
\mu^{*}(\pi) \geq \delta\left(\left[\nu^{\left(c_{\min }+1\right) / 2} \rho, \nu^{\left(c_{\max }-1\right) / 2} \rho\right]\right) \otimes \sigma^{\prime},
$$

for some irreducible representation $\sigma^{\prime}$.

Furthermore, by [17, Theorem 8.2], we see that there is some irreducible representation $\sigma^{\prime \prime}$ such that $\mu^{*}\left(\sigma_{d s}\right) \geq \delta\left(\left[\nu^{a} \rho, \nu^{\left(c_{\min }-1\right) / 2} \rho\right]\right) \otimes \sigma^{\prime \prime}$ and in the same way as before we deduce that

$$
\mu^{*}(\pi) \geq \delta\left(\left[\nu^{a} \rho, \nu^{\left(c_{\min }-1\right) / 2} \rho\right]\right) \otimes \pi^{\prime \prime}
$$

for some irreducible representation $\pi^{\prime \prime}$.

Suppose, contrary to our assumption, that $\pi$ is not a discrete series representation. Using a cuspidal support argument as before, we deduce that $\pi$ is a non-tempered representation and, using Lemma 3.1, we write it in the form $L\left(\delta_{1} \rtimes \tau\right)$, with $\delta_{1}=\delta\left(\left[\nu^{a_{1}} \rho_{1}, \nu^{b_{1}} \rho_{1}\right]\right), a_{1}+b_{1}<0$. In the same way as in the proof of Proposition 5.2, we deduce that $\rho_{1} \cong \rho, a-1 \leq b_{1} \leq b-1$ and $\sigma_{d s}$ is a subrepresentation of

$$
\delta\left(\left[\nu^{a} \rho, \nu^{b_{1}} \rho\right]\right) \times \delta\left(\left[\nu^{a_{1}} \rho, \nu^{b} \rho\right]\right) \rtimes \tau .
$$

Again, there are two possibilities to consider:

- $b_{1}>a-1$.

This shows that $\left(2 b_{1}+1, \rho\right) \in$ Jord' $^{\prime}$. Thus, $2 b_{1}+1$ equals either $c_{\max }$ or $c_{\min }$. If $2 b_{1}+1=c_{\max }$, then we have $a_{1}<-\left(c_{\max }-1\right) / 2$ and, since

$$
\delta\left(\left[\nu^{a} \rho, \nu^{b_{1}} \rho\right]\right) \times \delta\left(\left[\nu^{a_{1}} \rho, \nu^{b} \rho\right]\right) \cong \delta\left(\left[\nu^{a_{1}} \rho, \nu^{b} \rho\right]\right) \times \delta\left(\left[\nu^{a} \rho, \nu^{b_{1}} \rho\right]\right),
$$

we obtain that there is some irreducible representation $\sigma^{\prime}$ such that $\mu^{*}\left(\sigma_{d s}\right) \geq$ $\delta\left(\left[\nu^{-\left(c_{\max }+1\right) / 2} \rho, \nu^{b} \rho\right]\right) \otimes \sigma^{\prime}$. But, since in $\operatorname{Jord}^{\prime}$ we have $(2 b+1)_{-}=c_{\max }$, we get a contradiction with [9, Section 3$]$.

It remains to consider the case $2 b_{1}+1=c_{\text {min }}$.

Let us first show $a_{1}=-\left(c_{\max }-1\right) / 2$. Applying the structural formula for $\mu^{*}$ to (5.4), we obtain that there exist $i_{1}$ and $j_{1}$ such that $-\left(c_{\max }+1\right) / 2 \leq$ $i_{1} \leq j_{1} \leq b$ and $\pi^{\prime} \otimes \sigma_{s p}^{(1)} \leq \mu^{*}\left(\sigma_{s p}^{\prime \prime}\right)$ such that

$$
\begin{aligned}
& \delta\left(\left[\nu^{a_{1}} \rho, \nu^{b} \rho\right]\right) \times \delta\left(\left[\nu^{a} \rho, \nu^{\left(c_{\min }-1\right) / 2} \rho\right]\right) \\
& \quad \leq \delta\left(\left[\nu^{-i_{1}} \rho, \nu^{\left(c_{\max }-1\right) / 2} \rho\right]\right) \times \delta\left(\left[\nu^{j_{1}+1} \rho, \nu^{b} \rho\right]\right) \times \pi^{\prime}
\end{aligned}
$$

and

$$
\tau \leq \delta\left(\left[\nu^{i_{1}+1} \rho, \nu^{j_{1}} \rho\right]\right) \rtimes \sigma_{s p}^{(1)} .
$$


From the definition of $\sigma_{s p}^{\prime \prime}$, we deduce that $\pi^{\prime} \cong \delta\left(\left[\nu^{a} \rho, \nu^{\left(c_{\min }-1\right) / 2} \rho\right]\right)$ and $\left(i_{1}, j_{1}\right) \in\left\{\left(-\left(c_{\max }+1\right) / 2, a_{1}-1\right),\left(-a_{1},\left(c_{\max }-1\right) / 2\right)\right\}$. Furthermore, this implies

$$
\tau \leq \delta\left(\left[\nu^{-a_{1}+1} \rho, \nu^{\left(c_{\max }-1\right) / 2} \rho\right]\right) \rtimes \sigma_{s p}^{(1)},
$$

where, by [6, Theorem 4.6], $\sigma_{s p}^{(1)}$ is the strongly positive discrete series such that $\operatorname{Jord}\left(\sigma_{s p}^{(1)}\right)=\operatorname{Jord}\left(\sigma_{s p}\right) \backslash\left\{\left(c_{\max }, \rho\right)\right\} \cup\{(2 a-1, \rho)\}$.

Since $a_{1}<-\left(c_{\min }-1\right) / 2$, the representation

$$
\delta\left(\left[\nu^{-a_{1}+1} \rho, \nu^{\left(c_{\max }-1\right) / 2} \rho\right]\right) \rtimes \sigma_{s p}^{(1)}
$$

contains a tempered subquotient only if $-a_{1}+1>\left(c_{\max }-1\right) / 2$, i.e., if $a_{1}=$ $-\left(c_{\max }-1\right) / 2$. This gives

$$
\pi \cong L\left(\delta\left(\left[\nu^{-\left(c_{\max }-1\right) / 2} \rho, \nu^{\left(c_{\min }-1\right) / 2} \rho\right]\right) \rtimes \sigma_{s p}^{(1)}\right) .
$$

It follows from $[10$, Corollary 4.7$]$ that $\mu^{*}\left(\delta\left(\left[\nu^{-\left(c_{\min }-1\right) / 2} \rho, \nu^{\left(c_{\max }-1\right) / 2} \rho\right]\right) \rtimes\right.$ $\left.\sigma_{s p}^{(1)}\right)$ contains exactly two irreducible constituents of the form $\delta\left(\left[\nu^{\left(c_{\min }+1\right) / 2} \rho\right.\right.$, $\left.\left.\nu^{\left(c_{\max }-1\right) / 2} \rho\right]\right) \otimes \sigma^{\prime}$ and, by [10, Section 9], none of them is contained in

$$
\mu^{*}\left(L\left(\delta\left(\left[\nu^{-\left(c_{\max }-1\right) / 2} \rho, \nu^{\left(c_{\min }-1\right) / 2} \rho\right]\right) \rtimes \sigma_{s p}^{(1)}\right)\right) .
$$

But, this contradicts (5.6).

- $b_{1}=a-1$.

This clearly implies $\sigma_{d s} \hookrightarrow \delta\left(\left[\nu^{a_{1}} \rho, \nu^{b} \rho\right]\right) \rtimes \tau$ and Frobenius reciprocity shows that $\mu^{*}\left(\sigma_{d s}\right) \geq \delta\left(\left[\nu^{a_{1}} \rho, \nu^{b} \rho\right]\right) \otimes \tau$. Since $a_{1}<0$, Lemma 2.1, applied to (5.4), implies that the tempered representation $\tau$ appears as an irreducible subquotient of the induced representation

$$
\delta\left(\left[\nu^{-a_{1}+1} \rho, \nu^{\left(c_{\max }-1\right) / 2} \rho\right]\right) \rtimes \sigma_{s p}^{\prime \prime} .
$$

From [11, Proposition 3.1], we get that either $a_{1}=-\left(c_{\max }-1\right) / 2$ or $2\left(-a_{1}\right)+$ $1 \in \operatorname{Jord}_{\rho}\left(\sigma_{s p}^{\prime \prime}\right)$ and $\left\langle 2\left(-a_{1}\right)+1, c_{\text {max }}\right\rangle \cap \operatorname{Jord}_{\rho}\left(\sigma_{s p}^{\prime \prime}\right)=\emptyset$. It follows directly that $a_{1} \in\left\{-\left(c_{\max }-1\right) / 2,-\left(c_{\min }-1\right) / 2\right\}$. Furthermore, if $a_{1}=-\left(c_{\max }-1\right) / 2$ then $\tau \cong \sigma_{s p}^{\prime \prime}$, while $a_{1}=-\left(c_{\min }-1\right) / 2$ gives $\tau \cong \sigma_{s p}$.

In the first case we have $\pi \cong L\left(\delta\left(\left[\nu^{-\left(c_{\max }-1\right) / 2} \rho, \nu^{a-1} \rho\right]\right) \rtimes \sigma_{s p}^{\prime \prime}\right)$. By Proposition 3.2 , in $R(G)$ we have

$$
\begin{aligned}
\delta\left(\left[\nu^{-a+1} \rho, \nu^{\left(c_{\max }-1\right) / 2} \rho\right]\right) \rtimes \sigma_{s p}^{\prime \prime}= & \pi+\sigma+L\left(\delta\left(\left[\nu^{-\left(c_{\min }-1\right) / 2} \rho, \nu^{a-1} \rho\right]\right) \rtimes \sigma_{s p}\right) \\
& +L\left(\delta\left(\left[\nu^{-\left(c_{\max }-1\right) / 2} \rho, \nu^{\left(c_{\min }-1\right) / 2} \rho\right]\right) \rtimes \sigma_{s p}^{(1)}\right) .
\end{aligned}
$$

Let us now determine all irreducible constituents of the form $\delta\left(\left[\nu^{-a+1} \rho\right.\right.$, $\left.\left.\nu^{\left(c_{\max }-1\right) / 2} \rho\right]\right) \otimes \pi^{\prime}$ appearing in $\mu^{*}\left(\delta\left(\left[\nu^{-a+1} \rho, \nu^{\left(c_{\max }-1\right) / 2} \rho\right]\right) \rtimes \sigma_{s p}^{\prime \prime}\right)$, together with their multiplicities.

From Lemma 2.1 we get that there exist $i$ and $j$ such that $-a \leq i \leq j \leq$ $\left(c_{\max }-1\right) / 2$ and $\delta^{\prime} \otimes \sigma^{\prime} \leq \mu^{*}\left(\sigma_{s p}^{\prime \prime}\right)$ such that

$$
\delta\left(\left[\nu^{-a+1} \rho, \nu^{\left(c_{\max }-1\right) / 2} \rho\right]\right) \leq \delta\left(\left[\nu^{-i} \rho, \nu^{a-1} \rho\right]\right) \times \delta\left(\left[\nu^{j+1} \rho, \nu^{\left(c_{\max }-1\right) / 2} \rho\right]\right) \times \delta^{\prime}
$$


and

$$
\pi^{\prime} \leq \delta\left(\left[\nu^{i+1} \rho, \nu^{j} \rho\right]\right) \rtimes \sigma^{\prime} .
$$

Obviously, either $-i=-a+1$ or $j+1=-a+1$. If $j+1=-a+1$, then $\pi^{\prime}=\sigma_{s p}^{\prime \prime}$. If $-i=-a+1$ and $j+1=a$, again we obtain that $\pi^{\prime}=\sigma_{s p}^{\prime \prime}$. It remains to consider the case $-i=-a+1$ and $j+1>a$. Then we have $\delta^{\prime} \cong \delta\left(\left[\nu^{a} \rho, \nu^{j} \rho\right]\right)$ and $2 j+1 \in \operatorname{Jord}_{\rho}\left(\sigma_{s p}^{\prime \prime}\right)$. Since $a \leq j \leq\left(c_{\max }-1\right) / 2$, it follows that $j=\left(c_{\min }-1\right) / 2$ and, using [6, Theorem 4.6], we obtain that $\pi^{\prime} \leq \delta\left(\left[\nu^{a} \rho, \nu^{\left(c_{\min }-1\right) / 2} \rho\right]\right) \rtimes \sigma_{s p}^{(1)}$. By [11, Proposition 3.1], in $R(G)$ we have

$$
\delta\left(\left[\nu^{a} \rho, \nu^{\left(c_{\min }-1\right) / 2} \rho\right]\right) \rtimes \sigma_{s p}^{(1)}=L\left(\delta\left(\left[\nu^{-\left(c_{\min }-1\right) / 2} \rho, \nu^{-a} \rho\right]\right) \rtimes \sigma_{s p}^{(1)}\right)+\sigma_{s p}^{\prime \prime} .
$$

We have already seen that the only irreducible constituents of the form $\delta\left(\left[\nu^{-a+1} \rho, \nu^{\left(c_{\max }-1\right) / 2} \rho\right]\right) \otimes \pi^{\prime}$ appearing in $\mu^{*}\left(\delta\left(\left[\nu^{-a+1} \rho, \nu^{\left(c_{\max }-1\right) / 2} \rho\right]\right) \rtimes \sigma_{s p}^{\prime \prime}\right)$ are $\delta\left(\left[\nu^{-a+1} \rho, \nu^{\left(c_{\max }-1\right) / 2} \rho\right]\right) \otimes \sigma_{s p}^{\prime \prime}$ (and it appears there with multiplicity three) and

$$
\delta\left(\left[\nu^{-a+1} \rho, \nu^{\left(c_{\max }-1\right) / 2} \rho\right]\right) \otimes L\left(\delta\left(\left[\nu^{-\left(c_{\min }-1\right) / 2} \rho, \nu^{-a} \rho\right]\right) \rtimes \sigma_{s p}^{(1)}\right)
$$

(and it appears there with multiplicity one).

On the other hand, $\sigma$ and $L\left(\delta\left(\left[\nu^{-\left(c_{\min }-1\right) / 2} \rho, \nu^{a-1} \rho\right]\right) \rtimes \sigma_{s p}\right)$ are irreducible subquotients of $\delta\left(\left[\nu^{-a+1} \rho, \nu^{\left(c_{\min }-1\right) / 2} \rho\right]\right) \rtimes \sigma_{s p}$. Let us also determine all irreducible constituents of the form $\delta\left(\left[\nu^{-a+1} \rho, \nu^{\left(c_{\max }-1\right) / 2} \rho\right]\right) \otimes \pi^{\prime}$ appearing in $\mu^{*}\left(\delta\left(\left[\nu^{-a+1} \rho, \nu^{\left(c_{\min }-1\right) / 2} \rho\right]\right) \rtimes \sigma_{s p}\right)$. Similarly as before, there exist $i, j$ such that $-a \leq i \leq j \leq\left(c_{\min }-1\right) / 2$, and $\delta^{\prime} \otimes \sigma^{\prime} \leq \mu^{*}\left(\sigma_{s p}\right)$ such that

$$
\delta\left(\left[\nu^{-a+1} \rho, \nu^{\left(c_{\max }-1\right) / 2} \rho\right]\right) \leq \delta\left(\left[\nu^{-i} \rho, \nu^{a-1} \rho\right]\right) \times \delta\left(\left[\nu^{j+1} \rho, \nu^{\left(c_{\min }-1\right) / 2} \rho\right]\right) \times \delta^{\prime}
$$

and

$$
\pi^{\prime} \leq \delta\left(\left[\nu^{i+1} \rho, \nu^{j} \rho\right]\right) \rtimes \sigma^{\prime} .
$$

Since $\sigma_{s p}$ is strongly positive, [6, Theorem 4.6] shows that either $-i=-a+1$ or $j+1=-a+1$.

If $j+1=-a+1$, then $i=j, \delta^{\prime} \cong \delta\left(\left[\nu^{\left(c_{\min }+1\right) / 2} \rho, \nu^{\left(c_{\max }-1\right) / 2} \rho\right]\right)$ and $\pi^{\prime}=\sigma_{s p}^{\prime \prime}$. Assumptions $-i=-a+1$ and $j=a-1$ again give $\delta^{\prime} \cong \delta\left(\left[\nu^{\left(c_{\min }+1\right) / 2} \rho, \nu^{\left(c_{\max }-1\right) / 2} \rho\right]\right)$ and $\pi^{\prime}=\sigma_{s p}^{\prime \prime}$. Finally, if $-i=-a+1$ and $j>a-1$, using [6, Theorem 4.6] again, together with the description of the strongly positive representation $\sigma_{s p}$, we deduce that $j=\left(c_{\min }-1\right) / 2$, $\delta^{\prime} \cong \delta\left(\left[\nu^{a} \rho, \nu^{\left(c_{\max }-1\right) / 2} \rho\right]\right)$ and $\pi^{\prime} \leq \delta\left(\left[\nu^{a} \rho, \nu^{\left(c_{\min }-1\right) / 2} \rho\right]\right) \rtimes \sigma_{s p}^{(1)}$.

Consequently, the only irreducible constituents of the form

$$
\delta\left(\left[\nu^{-a+1} \rho, \nu^{\left(c_{\max }-1\right) / 2} \rho\right]\right) \otimes \pi^{\prime}
$$

appearing in $\mu^{*}\left(\delta\left(\left[\nu^{-a+1} \rho, \nu^{\left(c_{\min }-1\right) / 2} \rho\right]\right) \rtimes \sigma_{s p}\right)$ are $\delta\left(\left[\nu^{-a+1} \rho, \nu^{\left(c_{\max }-1\right) / 2} \rho\right]\right) \otimes$ $\sigma_{s p}^{\prime \prime}$ (and it appears there with multiplicity three) and

$$
\delta\left(\left[\nu^{-a+1} \rho, \nu^{\left(c_{\max }-1\right) / 2} \rho\right]\right) \otimes L\left(\delta\left(\left[\nu^{-\left(c_{\min }-1\right) / 2} \rho, \nu^{-a} \rho\right]\right) \rtimes \sigma_{s p}^{(1)}\right)
$$


(and it appears there with multiplicity one).

It follows from [11, Theorem 2.1] that $\delta\left(\left[\nu^{-a+1} \rho, \nu^{\left(c_{\min }-1\right) / 2} \rho\right]\right) \rtimes \sigma_{s p}$ is a length three representation, and we denote its discrete series subrepresentation different than $\sigma$ by $\sigma_{1}$. Also, we denote the admissible triple attached to $\sigma_{1}$ by (Jord, $\sigma_{\text {cusp }}, \epsilon_{1}$ ). Since $\sigma \not \sigma_{1}$, it follows that $\epsilon \neq \epsilon_{1}$ and, consequently, $\epsilon_{1}\left(\left(c_{\min }, \rho\right),\left(c_{\max }, \rho\right)\right)=-1$. Using [17, Proposition 7.2], we conclude that $\mu^{*}\left(\sigma_{1}\right)$ does not contain an irreducible constituent of the form $\delta\left(\left[\nu^{-a+1} \rho, \nu^{\left(c_{\max }-1\right) / 2} \rho\right]\right) \otimes \pi^{\prime}$.

It follows directly from Proposition 3.2 that both representations $\sigma$ and $L\left(\delta\left(\left[\nu^{-\left(c_{\min }-1\right) / 2} \rho, \nu^{a-1} \rho\right]\right) \rtimes \sigma_{s p}\right)$ are contained in $\delta\left(\left[\nu^{-a+1} \rho, \nu^{\left(c_{\max }-1\right) / 2} \rho\right]\right) \rtimes$ $\sigma_{s p}^{\prime \prime}$. Now the calculation of $\mu^{*}\left(\delta\left(\left[\nu^{-a+1} \rho, \nu^{\left(c_{\max }-1\right) / 2} \rho\right]\right) \rtimes \sigma_{s p}^{\prime \prime}\right)$, based on Lemma 2.1, implies that

$$
\mu^{*}\left(L\left(\delta\left(\left[\nu^{-\left(c_{\max }-1\right) / 2} \rho, \nu^{a-1} \rho\right]\right) \rtimes \sigma_{s p}^{\prime \prime}\right)\right)
$$

does not contain an irreducible constituent of the form

$$
\delta\left(\left[\nu^{-a+1} \rho, \nu^{\left(c_{\max }-1\right) / 2} \rho\right]\right) \otimes \pi^{\prime},
$$

contradicting (5.5).

In the second case we have $\pi \cong L\left(\delta\left(\left[\nu^{-\left(c_{\min }-1\right) / 2} \rho, \nu^{a-1} \rho\right]\right) \rtimes \sigma_{s p}\right)$. We have already seen that $\mu^{*}\left(\delta\left(\left[\nu^{-a+1} \rho, \nu^{\left(c_{\min }-1\right) / 2} \rho\right]\right) \rtimes \sigma_{s p}\right)$ has exactly two irreducible constituents of the form $\delta\left(\left[\nu^{a} \rho, \nu^{\left(c_{\min }-1\right) / 2} \rho\right]\right) \otimes \pi^{\prime}$ and none of them is contained in $\mu^{*}\left(L\left(\delta\left(\left[\nu^{-\left(c_{\min }-1\right) / 2} \rho, \nu^{a-1} \rho\right]\right) \rtimes \sigma_{s p}\right)\right.$, contrary to (5.7).

Consequently, $\pi$ is a discrete series representation. Let us denote its corresponding admissible triple by $\left(\operatorname{Jord}^{\prime \prime}, \sigma_{\text {cusp }}, \epsilon^{\prime \prime}\right)$. It directly follows that Jord" $^{\prime \prime}=$ Jord. Using [17, Proposition 7.2], together with (5.6), we obtain that $\epsilon^{\prime \prime}\left(\left(c_{\min }, \rho\right),\left(c_{\max }, \rho\right)\right)=1$. Also, [17, Proposition 7.2] and (5.7) imply $\epsilon^{\prime \prime}\left((2 a-1, \rho),\left(c_{\min }, \rho\right)\right)=1$. Properties of the $\epsilon$-function imply that $\epsilon^{\prime \prime}$ equals -1 on all other pairs. Therefore, $\epsilon^{\prime \prime}=\epsilon$ and, consequently, $\pi \cong \sigma$. Thus, $\sigma_{d s}$ is a subrepresentation of $\delta \rtimes \sigma$ and the proposition is proved.

We emphasize that from the last part of the proof of Proposition 5.3 is easy to deduce that a discrete series subquotient of $\delta \rtimes \sigma$ different than $\sigma_{d s}$, which exists by Proposition 4.5, is not a subrepresentation.

The previous sequence of propositions, together with results obtained in the previous section, implies the following result:

Theorem 5.4. Positive generalized principal series contains a discrete series subquotient if and only if it contains a discrete series subrepresentation.

ACKNowledgements.

We would like to thank Marko Tadić for useful conversations regarding the Jacquet modules of discrete series representations and to Goran Muic for his active interest in the publication of this paper. We would also like to thank referees for a number of corrections and very useful suggestions. 


\section{REFERENCES}

[1] J. Arthur, The endoscopic classification of representations. Orthogonal and symplectic groups, American Mathematical Society, Providence, 2013.

[2] I. N. Bernstein and A. V. Zelevinsky, Induced representations of reductive p-adic groups. I, Ann. Sci. École Norm. Sup. (4) 10 (1977), 441-472.

[3] M. Hanzer, The generalized injectivity conjecture for classical p-adic groups, Int. Math. Res. Not. IMRN (2010), 2010, 195-237.

[4] I. Matić, Strongly positive representations of metaplectic groups, J. Algebra 334 (2011), $255-274$.

[5] I. Matić, The conservation relation for discrete series representations of metaplectic groups, Int. Math. Res. Not. IMRN (2013), 2013, 5227-5269.

[6] I. Matić, Jacquet modules of strongly positive representations of the metaplectic group $\widehat{S p(n)}$, Trans. Amer. Math. Soc. 365 (2013), 2755-2778.

[7] I. Matić, On Jacquet modules of discrete series: the first inductive step, J. Lie Theory 26 (2016), 135-168.

[8] I. Matić and M. Tadić, On Jacquet modules of representations of segment type, Manuscripta Math. 147 (2015), 437-476.

[9] C. Mœglin, Sur la classification des séries discrètes des groupes classiques p-adiques: paramètres de Langlands et exhaustivité, J. Eur. Math. Soc. (JEMS) 4 (2002), 143-200.

[10] C. Møglin and M. Tadić, Construction of discrete series for classical p-adic groups, J. Amer. Math. Soc. 15 (2002), 715-786.

[11] G. Muić, Composition series of generalized principal series; the case of strongly positive discrete series, Israel J. Math. 140 (2004), 157-202.

[12] G. Muić, Howe correspondence for discrete series representations; the case of $(S p(n), O(V))$, J. Reine Angew. Math. 567 (2004), 99-150.

[13] G. Muić, Reducibility of generalized principal series, Canad. J. Math. 57 (2005), 616647.

[14] F. Shahidi, A proof of Langlands' conjecture on Plancherel measures; complementary series for p-adic groups, Ann. of Math. (2) 132 (1990), 273-330.

[15] F. Shahidi, Twisted endoscopy and reducibility of induced representations for $p$-adic groups, Duke Math. J. 66 (1992), 1-41.

[16] M. Tadić, Structure arising from induction and Jacquet modules of representations of classical p-adic groups, J. Algebra 177 (1995), 1-33.

[17] M. Tadić, On tempered and square integrable representations of classical p-adic groups, Sci. China Math. 56 (2013), 2273-2313.

[18] A. V. Zelevinsky, Induced representations of reductive p-adic groups. II. On irreducible representations of $G L(n)$, Ann. Sci. École Norm. Sup. (4) 13 (1980), 165-210.

I. Matić

Department of Mathematics

University of Osijek

Trg Ljudevita Gaja 6, 31000 Osijek

Croatia

E-mail: imatic@mathos.hr

Received: 30.3.2015. 